\title{
Intelligent Bearing Fault Diagnosis using Swarm Decomposition Method and New Hybrid Particle Swarm Optimization Algorithm
}

Saeed Nezamivand Chegini ( $\nabla$ saeed.nezamivand@gmail.com )

University of Guilan https://orcid.org/0000-0002-0744-7919

Pouriya Amini

Technische Universitat Dresden

Bahman Ahmadi

University of Kurdistan

Ahmad Bagheri

University of Guilan

Illia Amirmostofian

University of Guilan

\section{Research Article}

Keywords: Bearing Fault Diagnosis, Swarm Decomposition, Optimized Compensation Distance Evaluation, Hybrid Particle Swarm Optimization Algorithm, Support Vector Machine

Posted Date: August 30th, 2021

DOI: https://doi.org/10.21203/rs.3.rs-836230/v1

License: (c) (i) This work is licensed under a Creative Commons Attribution 4.0 International License.

Read Full License 


\title{
Intelligent Bearing Fault Diagnosis using Swarm Decomposition Method and New Hybrid Particle Swarm Optimization Algorithm
}

\author{
Saeed Nezamivand Chegini ${ }^{*}$, Pouriya Amini ${ }^{2}$, Bahman Ahmadi ${ }^{3}$, Ahmad Bagheri ${ }^{1}$, Illia Amirmostofian ${ }^{1}$ \\ 1- Department of Dynamics, Control, and Vibrations, Faculty of Mechanical Engineering, University \\ of Guilan, Rasht, Iran. \\ 2- Institute of Acoustics and Speech Communication, TU Dresden, Germany \\ 3- Department of Mechanical Engineering, Faculty of Engineering, University of Kurdistan, \\ Sanandaj, Iran \\ * saeed.nezamivand@gmail.com
}

\begin{abstract}
The quality of information extracted from the vibration signals, and the accuracy of the bearing status detection depend on the methods used to process the signal and select the informative features. In this paper, a new hybrid approach is introduced in which the relatively new Swarm Decomposition (SWD) method and the optimized Compensation Distance Evaluation Technique (OCDET) are used to enhance the signal processing stage and to improve the optimal features selection process, respectively. Firstly, the vibration signals are decomposed into their Oscillatory Components (OCs) using the SWD. The feature matrix is constructed by computing the time-domain features for the OCs. The CDET method is consequently utilized to select the most sensitive features corresponding to the bearing status. On the other hand, The CDET approach contains a parameter called threshold which affects the number of the selected features. In this way, the hybrid optimization algorithm, which is a combination of the Particle Swarm Optimization (PSO) algorithm with the Sine-Cosine Algorithm (SCA) and the Levy flight distribution, has been used to select the optimal CDET threshold and improve the Support Vector Machine (SVM) classifier. The proposed technique ability is evaluated by vibration signals corresponding to different bearing defects and various speeds. The results indicate the capability of the proposed fault diagnosis method in identifying the very small-size defects under various bearing conditions. Finally, the presented method shows better performance in comparison with other well-known methods in the most of the case studies.
\end{abstract}

Keywords: Bearing Fault Diagnosis; Swarm Decomposition; Optimized Compensation Distance Evaluation; Hybrid Particle Swarm Optimization Algorithm; Support Vector Machine 


\section{Introduction}

Mechanical equipment holds are the basics in modern industry with the rapid improvements in science and technology. Among these, rotating machinery is considered as one of the most complex and functional equipment in industrial applications. Thus, it seems essential to diagnosis the faults of rotary machines as one of the most important parts of design and maintenance in a system (Liu et al. 2018). The technique of fault diagnosis (FD) of rotating machinery consists of four functions, namely, fault identification, fault isolation, fault detection, and fault estimation. In other words, the FD means the determination of kind, size, location, and time of occurrence of a fault. Besides, with a similar point of view, one can divide the FD process into three distinguished parts: 1) inspecting the function of equipment 2) determining the existence and the reason for failure, and 3) predicting the fault development (Jardine et al. 2006).

Intelligent fault diagnosis (IFD) methods include four main parts: signal processing, feature extraction (FE), feature selection (FS), and pattern recognition. The signal processing technique plays a crucial role in extracting useful information from the vibration data. In recent decades, various methods have been introduced for the analysis of vibration signals, including the Wavelet Transforms (WT) (Daubechies 1990), Empirical Mode Decomposition (EMD) (Huang et al. 1998), Ensemble EMD (EEMD) (Wu and Huang, 2009), Complete EEMD with Adaptive Noise (CEEMDAN) (Torres et al. 2011), and Empirical Wavelet Transform (EWT) (Gilles 2013). Generally, the status of the rotating machinery cannot be distinguished only through the signal processing methods and extracting various features. Due to the complicated nature of vibration data, in practical applications, the most appropriate features for monitoring the condition of the machine cannot be identified in advance. Therefore, in various researches, the FS methods such as filter method, wrapper method, and hybrid method had been used. Summary descriptions of some of the researches conducted in recent years on intelligent troubleshooting methods are presented following:

Chen et al. (2016) indicated the important role of WT in rotating machinery fault diagnosis (RMFD) by some simulation and field test experiments. Ali et al. (2015) classified and characterized the vibration signals of the damaged bearing by EMD, and suggested a new mathematical model for selecting the most significant intrinsic mode functions (IMFs). Deng et al. (2017) proposed a new integrating intelligent method based on EMD, fuzzy entropy, Particle Swarm Optimization (PSO), and Support Vector Machine (SVM) to verify the efficiency of the presented technique for the FD of a motor bearing. To enhance the fault detection accuracy of incipient fault in rolling bearing, Gao et al. (2018) investigated an 
intelligent fault detection scheme combined with EEMD, Principle Component Analysis (PCA), and Probabilistic Neural Network (PNN). Nezamivand Chegini et al. (2019a) suggested a novel hybrid vibration signal denoising method using the EWT technique. The authors also used the kurtosis parameter and the envelope spectrum (ES) of the denoised bearing vibration signals at different shaft speeds to detect the early fault as well as to diagnose the type of fault. In another work (Nezamivand Chegini et al. 2019b), the authors presented a new approach for selecting fault-sensitive features. They utilized the statistical time-domain features, the Wavelet Packet Transform (WPT), and EMD techniques to extract a large number of features from the vibration signals. In this way, the most appropriate features were determined using the FDAF-score technique, the Binary PSO (BPSO) algorithm, and the SVM. Ding et al. (2020a) applied the Genetic Mutation Particle Swarm Optimization (GMPSO) algorithm for improving the VMD method. They employed the features extracted from the improved VMD components as the PNN inputs to diagnosis the gear status. In another work (Ding et al. 2020b), the authors determined the ES of the IMFs obtained by the GMPSO-VMD approach for bearing fault detection. Wei et al. (2020) introduced a new imbalanced FD framework based on Cluster-Majority Weighted Minority Oversampling Technique (Cluster-MWMOTE) and a Moth-Flame Optimization (MFO)based Least Squares SVM (LS-SVM) to address the FD of rolling bearing under complex conditions. Shao et al. (2021) proposed an improved the FE method by Integrating VMD (IVMD) and Time-Shift Multiscale Dispersion Entropy (TSMDE). They introduced a novel algorithm called the Vibrational Harris Hawks Optimization (VHHO) algorithm for selecting the optimal SVM parameters. Zhang et al. (2021) suggested a new methodology based on improved Symplectic Geometry Mode Decomposition (SGMD) and optimized SVM. The authors utilized the SVM optimized by Inter-Cluster Distance in Feature Space (ICDTS) and the $\mathrm{HHO}$ algorithm to diagnose faults.

The most important phase of the IFD techniques is the processing of vibration signals. In most of the above studies, in the signal processing phase, WT, EMD, EEMD, EWT, and VMD techniques were used to extract the informative components. The mode mixing phenomenon, end effects, and the lack of an adequate mathematical theory are among the weaknesses of EMD. However, each of these techniques suffers from deficiencies. Determining the added noise amplitude, the number of ensemble trials and computational complexity are limitations of the EEMD method. Selecting the appropriate wavelet base function and the number of decomposition levels is the WT challenge. The segmentation of the signal Fourier spectrum in the EWT method, and predetermining the key parameters of 
the VMD method are among other limitations. On the other hand, the FS methods and the pattern recognition techniques usually have parameters that how they are set directly affects the accuracy of intelligent troubleshooting approaches. However, in some studies, metaheuristic optimization algorithms were used to determine the optimal value of these parameters. Due to the weakness of these algorithms in determining the global solution, in most cases, the desired results for the optimal parameters cannot be achieved.

Given the above challenges, the following general objectives have been considered in the present study:

- Applying a suitable signal processing method to extract the informative components from the vibration signals

- Improving the FS and fault classifier steps using a powerful optimization algorithm In this paper, a novel intelligent multi-FD approach is introduced. In the proposed technique, for the first time, the Swarm Decomposition (SWD) method recently introduced by Apostolidis and Hadjileontiadis (2017) is used to decompose the vibration data. Inspired by swarm intelligence and based on the Swarm Filtering $(\mathrm{SwF})$ and the iterative algorithm, the SWD method had overcome the limitations of techniques such as EMD, EWT, and VMD (Apostolidis and Hadjileontiadis, 2017; Miao et al. 2019). Also, benefiting from the advantages of noise suppression and intelligent decomposition, the SWD provides efficient performance in solving the problem of mode mixing. In the proposed scheme, the Compensation Distance Evaluation Technique (CDET) and SVM techniques are employed to select the most informative fault-related features and predicting the status of machine components, respectively. On the other hand, the SVM inputs are the features that are selected by the CDET. It is worth mentioning that there is a parameter in the CDET that plays a significant role in the quality of the selected attributes. As the second innovation, the hybrid PSO algorithm called PSOSCALF (Nezamivand Chegini et al. 2018) is utilized for specifying the optimum values of the CDET threshold and the SVM parameters. The PSOSCALF algorithm is a combination of the velocity and position updating equations of PSO, the response updating equations of the Sine-Cosine Algorithm (SCA), and the Levy flight distribution. Nezamivand Chegini et al. (2018) showed that the PSOSCALF algorithm can solve the challenges of early convergence and falling in the local optimum. Finally, the optimized CDET (OCDET) is utilized to select the best feature set as the SVM inputs. The simulation signal and the experimental vibration data corresponding to the different bearings defects at different speeds have been used to investigate the capabilities of the proposed 
technique. The results of the simulated signal indicate that the SWD decomposition method outperforms the EMD, CEEMDAN, VMD techniques in forming the fault-related features. Also, the results of the vibration signals show that the performance of the proposed method is superior to other techniques.

The remainder of this article is organized as follows: In Section 2, the methods used in this article are briefly described. The proposed intelligent method is presented in Section 3. Section 4 describes the validation simulation of the method. The current work is examined by two vibration data set and results are interpreted in Section 5. Finally, the conclusion of the article is presented in Section 6.

\section{Methods}

\subsection{Swarm Decomposition Method}

The swarm decomposition method is a novel scheme to analyze the non-stationary and multicomponent signals. This technique is recently suggested based on the swarm intelligence concept (Apostolidis and Hadjileontiadis 2017). SWD decomposes a multi-component signal into some oscillatory components (OCs) by implementing the swarm filtering ( $\mathrm{SwF}$ ) principles. In the SWD model, the signal $x[n]$ is considered as the prey location at time step $n$ and denoted with $p_{\text {prey }}[n]$. The signal processing procedure is equivalent to hunting prey in a swarm manner. The swarm contains $M$ members, and each particle of the swarm is characterized by two parameters of position, $p_{i}[n]$, and velocity, $v_{i}[n]$.

The position and velocity of individuals in the swarm model are updated by forces produced among population members. These forces are classified into two major groups called the driving force and the cohesion force. The driving force $F_{D r, i}^{n}$ is the one that the prey implies to the $i$-th member of the swarm at the time step $n$ in the hunting process and is obtained as follows:

$F_{D r, i}^{n}=p_{\text {prey }}[n]-p_{i}[n-1]$

Unlike the driving force, the cohesion force, $F_{C o h, i}^{n}$, is the result of the interactions of all members of the population with the $i$-th member and is obtained by the following equations:

$$
\begin{aligned}
& F_{C o h, i}^{n}=\frac{1}{M-1} \sum_{\substack{j=1 \\
j \neq i}}^{M} f\left(p_{i}[n-1]-p_{j}[n-1]\right) \\
& f(d)=-\operatorname{sgn}(d) \ln \left(\frac{|d|}{d_{c r}}\right)
\end{aligned}
$$


In Eqs. (2) and (3), $\operatorname{sgn}($.$) and |. | stand for sign function and absolute value, respectively.$ The cohesion force between the $i$-th and $j$-th members can be applied as repulsive or attraction force and its type is determined by function $f(d)$. The parameter $d_{c r}$ is a key parameter for controlling the distribution of members in the population. This parameter corresponds to the crucial distance from which the force between two members of the swarm is zero, that is, $f\left(d_{c r}\right)=0$. In (Rao and Kumaresan 2000), the value of $d_{c r}$ is considered as the root mean square (RMS) of the input multi-component signal. The updating equations of the position of each member in the hunting process are as follows:

$v_{i}[n]=v_{i}[n-1]+\delta\left(F_{D r, i}^{n}+F_{C o h, i}^{n}\right)$

$p_{i}[n]=p_{i}[n-1]+\delta v_{i}[n]$

$\delta$ is one of the most important parameters in the SWD process. The parameter $\delta$ controls the flexibility of the swarm members. The output of SWD is considered as follows:

$y[n]=\beta \sum_{i=1}^{M} p_{i}[n]$

In fact, in each repetition, the output signal of $\mathrm{SwF}$ is equal to the weighted summation of the paths of all members. Adopted from (Rao and Kumaresan 2000), the value of parameter $\beta$ is considered as 0.005. Moreover, it is assumed that the initial value of the velocity of all members is zero and their initial positions are calculated as follows:

$p_{i}[0]=p_{\text {prey }}[n]+d_{c r}\left(i-1-\frac{M}{2}\right), \forall i=1,2, \ldots, M$

Let $S_{\text {multi }}[n]$ is a non-stationary multi-component signal composed of the monocomponent signals $S_{\text {mono,l }}[n]$, and $y_{M, \delta}[n]$ is the SwF output for the SWD key parameters, i.e., $\mathrm{M}$ and $\delta$. On the other hand, the swarm parameters $\mathrm{M}$ and $\delta$ affect the outputs of the $\mathrm{SwF}$ method. Therefore, by solving the minimization problem in Eq. (8) using the GA optimization algorithm, Eqs. (9) and (10) are obtained to calculate the parameters $\mathrm{M}$ and $\delta$ :

$$
\begin{aligned}
& \underset{\delta, M}{\arg \min } \sum_{k}\left\{\left|Y_{\delta, M}(k)\right|-S_{\text {multi }}(k)\right\}^{2} \\
& M(\bar{\omega})=\left[33.46 \bar{\omega}^{-0.735}-29.1\right] \\
& \delta(\bar{\omega})=-1.5 \bar{\omega}^{2}+3.454 \bar{\omega}-0.01
\end{aligned}
$$

where $Y_{\delta, M}(k)$ and $S_{m u l t i}(k)$ are the discrete Fourier transform of the signals $y_{M, \delta}[n]$ and $S_{\text {multi }}[n]$, respectively. Also, $\bar{\omega}$ denotes the normalized frequency. The repetitive method in the SWD approach is similar to the sifting process so that each OC is obtained by applying 
the $\mathrm{SwF}$ in each repetition. Then, the obtained OC is subtracted from the input signal. If the input signal includes no other OCs, the SWD approach is stopped. In the other words, the repetitive SWD process is terminated whenever the standard deviation of the results of the two successive iterations, i.e., $y_{i}[n-1]$ and $y_{i}[n]$ is less than the predetermined threshold of $S t D_{t h}$

At the beginning of the sifting process (i.e., $i t=0)$ it is assumed that $x_{0}[n]=y_{0}[n]=x[n]$. Then, the energy spectral density of $y_{0}[n]$, i.e., $S_{x_{i t}}(\omega)$, is calculated and the frequency with the highest amplitude in the energy spectrum smoothed by the Savitzky-Golay filter (SGfilter) is considered as the optimal mode frequency. Hence, the optimal frequency is estimated as follows:

$\omega_{o p t}=\underset{\omega}{\arg \max }\left(S_{x_{i t}}^{\prime}(\omega)>P_{t h}\right)$

$S_{x_{i t}}^{\prime}(\omega)=\operatorname{SGfilter}\left(S_{x_{i t}}(\omega)\right)$

where $\omega_{o p t}$ is the optimal frequency. The parameter $P_{t h}$ limits the search range to find the optimal frequency and considerably affects the computational time. In the next stage, the parameters $\mathrm{M}$ and $\delta$ are determined using Eqs. (9) and (10). Also, $y_{i}[n]$ is obtained from Eq. (4) by setting $i=1$. If the standard deviation of two successive sequences, i.e., $\sum \frac{\left|y_{i}[n]-y_{i-1}[n]\right|^{2}}{\left(y_{i-1}[n]\right)^{2}}$, is less than the predetermined threshold value of $S t D_{t h}$, the filtered signal $x_{i t}^{\prime}[n]$ is considered equal to the signal $y_{i}[n]$. Otherwise, in an iterative procedure, the index $i$ is increased by one unit and the signal $x_{i t}[n]$ is replaced with the signal $y_{i}[n]$. Finally, if $S_{x_{i t}}^{\prime}(\omega)<P_{t h}$, the residue signal $x_{i t+1}[n]$ is obtained by $x_{i t+1}[n]=x_{i t}[n]-x_{i t}^{\prime}[n]$, otherwise, $x_{i t+1}[n]=x_{i t}[n]$. The above iterative procedure continues until no more OCs are extracted. The flowchart of SWD is illustrated in Fig. 1.

In the SWD technique, the number of the extracted modes, and their quality depend on the parameters $S t D_{t h}$ and $P_{t h}$. In (Apostolidis and Hadjileontiadis 2017), the value of these parameters is set to 0.1 . 


\subsection{The FE Procedure}

The vibration signals of bearings have non-stationary, complex, and non-linear dynamics behavior. To provide usable information about the characteristics of faulty bearings, different FE methods are introduced. Moreover, to construct the feature vector corresponding to each signal, the following procedure is proposed:

1. Each vibration signal is decomposed into different OCs using the SWD method. There are two parameters $\left(P S_{t h}\right.$ and $\left.T_{t h}\right)$ which have a significant role in the SWD method and these parameters are considered as 0.1, adopted from (Apostolidis and Hadjileontiadis 2017).

2. The two OCs with the highest kurtosis value are selected to extract the features in the time domain.

3. For each OC, the statistical features listed in Table 1 are extracted (features FV1FV21 and FV22-FV42).

After extracting the statistical features, the feature vector corresponding to each vibration signal is constructed. The schematic of the feature vector elements is depicted in Fig. 2. The feature vectors of all vibration signals are calculated. Also, the general form of the new data set is obtained as follow:

$F M=\left\{q_{m, c, j}\right\}, \quad m=1,2, \ldots M_{c}, \quad c=1,2, \ldots, C, \quad j=1,2, \ldots, J$

where, $q_{m, c, j}$ stands for $j$ th feature value of $m$ th sample under $c$ th condition and $M_{c}$ stands for the sample number of $c$ th condition. Also, $J$ denotes the feature number of each sample. In this study, $J=42$ and $C$ is equal to the number of rotating machinery statuses. 


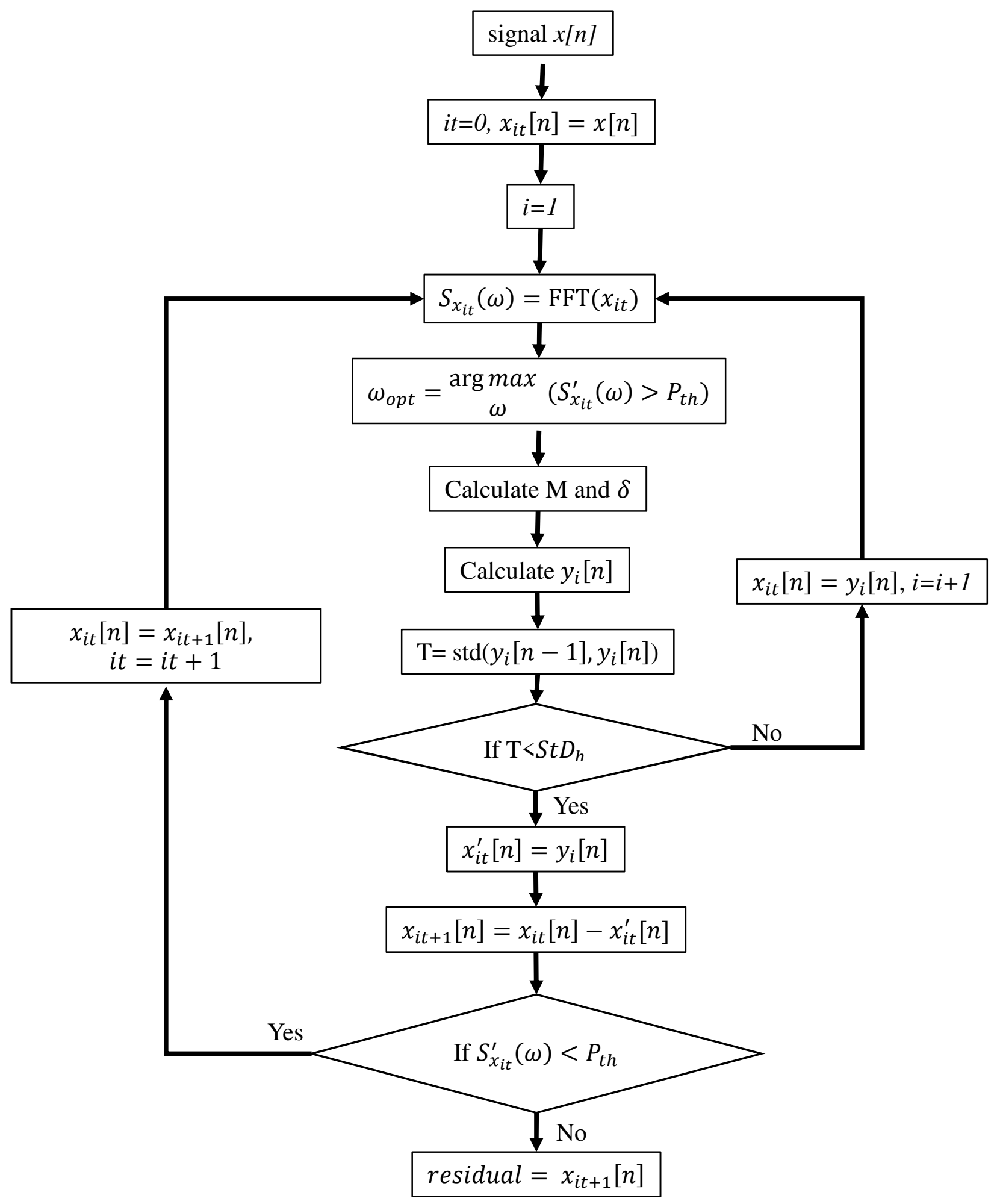

Fig. 1 The flowchart of the SWD decomposition method. 
Table 1 Time domain and frequency domain features (Zhang at al. 2018b).

\begin{tabular}{ll}
\hline Time-domain Features & Standard deviation $=\sqrt{\frac{1}{N-1} \sum_{i=1}^{N}\left(x_{i}-\bar{x}\right)^{2}}$ \\
\hline Mean $=\frac{1}{N} \sum_{i=1}^{N} x(i)$ & Skewness $=\frac{\frac{1}{N} \sum_{i=1}^{N}\left(x_{i}-\bar{x}\right)^{3}}{\left(\frac{1}{N} \sum_{i=1}^{N}\left(x_{i}-\bar{x}\right)^{2}\right)^{\frac{3}{2}}}$ \\
Maximum $=\max (x(i))$ & Kurtosis $=\frac{\frac{1}{N} \sum_{i=1}^{N}\left(x_{i}-\bar{x}\right)^{4}}{\left(\frac{1}{N} \sum_{i=1}^{N}\left(x_{i}-\bar{x}\right)^{2}\right)^{2}}$ \\
Minimum $=\min (x(i))$ & Crest factor $=\frac{\max (\text { abs }(x))}{R M S}$ \\
Median $=x\left(\frac{N+1}{2}\right)$ & Shape factor $=\frac{x_{R M S}}{\left(\frac{1}{N}\right) \sum_{i=1}^{N}\left|x_{i}\right|}$ \\
Energy $=\int|x(t)|^{2} d t$ & Tigear - Kaiser energy $\left.=\sum_{i=1}^{N}\left([x(i)]^{2}-x(i-1) x(i+1)\right]\right)$ \\
Entropy $=-\sum_{i=1}^{N} x_{i}^{2} \log \left(x_{i}^{2}\right)$ & Hybrid feature $2=\log ($ Kurtosis \\
Mearest factor $+\left(\frac{R M S}{0.078}\right)^{\text {Range }}$
\end{tabular}



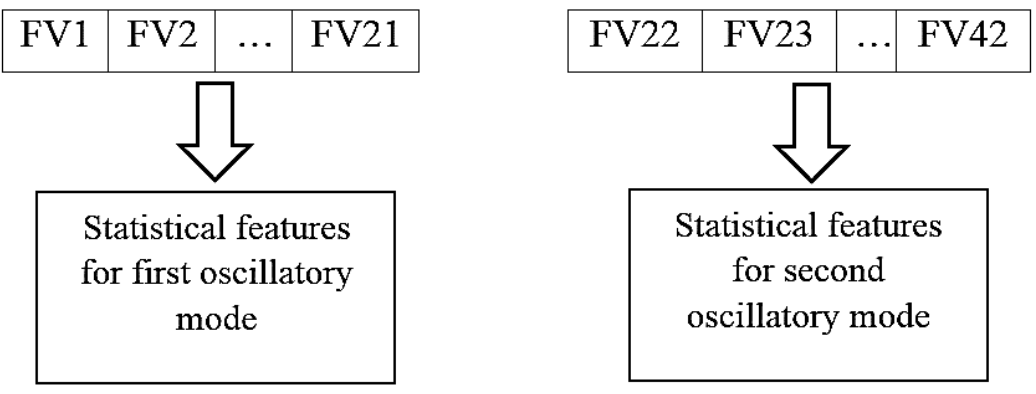

Fig. 2 The schematic of feature vector elements.

\subsection{The CDET Technique}

Generally, some features show their relevance to the faults, while others are not sensitive. To increase the diagnosis effectiveness as well as to decrease the curse of dimensionality problem, it is necessary to select and highlight the important features that contain the related information of the bearing. On the other hand, redundant or irrelevant features should be weakened and thrown away. The CDET, which is the improved version of feature selection by distance evaluation method, has been introduced in the literature (Yang and Kim 2006, Yang et al. 2004, Widodo and Yang 2007, Hu et al. 2007, and Lei et al. 2008). The FS scheme based on CDET is presented in Appendix A. According to Appendix A, the CDET assigns a weight to each feature. Then a predetermined parameter, denoted by $\xi$, is considered. The features with weights more than the $\xi$ threshold are selected as desirable features, otherwise, they are removed.

In some studies, e.g., (Lei et al. 2008), the value of $\xi$ is determined experimentally for selecting the informative features. Selecting the most appropriate value for $\xi$ is the main challenge in the implementation of the CDET procedure. Considering an inefficient value for $\xi$ leads to remove the useful features and remain the irrelevant ones. In the proposed intelligent method, a novel approach is presented for identifying the optimal value for $\xi$ based on the PSOSCALF algorithm and improvement of the FD accuracy.

\subsection{The PSOSCALF Optimization Algorithm}

The particle swarm optimization technique (PSO) is a population-based computational algorithm that had been widely used in many recent applications. Each of the members of the swarm called particle is determined by two vectors the position and velocity in the search space in the optimization procedure. Since the start of the optimization procedure, the particles with the position and velocity vectors are randomly generated in the design space. Then, in each replication of the implementation of the PSO algorithm, each particle updates 
its position in the search space based on its best personal experience, and the best experience of the whole particles.

Despite its simplicity in implementation, the PSO algorithm has some disadvantages e.g., premature convergence and falling at local optimum (Shi 2001, Amini Digehsara et al. 2019, Amini Digehsara et al. 2020). More recently, in 2018, Nezamivand Chegini et al. (2018) presented the PSOSCALF approach to resolve these points of weakness. In the PSOSCALF algorithm, the position updating equations in the SCA optimization algorithm are combined with the Levy flight distribution. Then, by utilizing these hybrid relationships in the PSO technique, both exploration and exploitation phases are strengthened as the optimization procedure is performed. Levy flight can create the long jump of the particles in the search space. This ability provides a more efficient search in the search area and leads to produce the variant particles throughout the optimization procedure. The SCA algorithm transits smoothly from exploration to exploitation using several random and adaptive variables and changing the range of the sine and cosine functions. The equations of the PSOSCALF have presented in Appendix B.

Nezamivand Chegini et al. (2018) evaluated the PSOSCALF algorithm in optimizing different benchmark functions and various nonlinear constrained engineering problems. Then, they compared the results with many algorithms of the PSO family and other algorithms. The results indicated that the PSOSCALF algorithm has better performance than other algorithms in finding the global minimum of the optimization problems. Due to these reasons, in the present work, the PSOSCALF optimization algorithm is used to determine the optimal parameters of the CDET technique and the SVM classifier.

\subsection{The SVM Method}

Support Vector Machine is a computational learning algorithm to classify small samples (Yang et al. 2004). The SVM builds an optimal separating hyperplane $f(x)=0$ between data sets. In other words, SVM solves a constrained quadratic optimization problem on the basis of the Structural Risk Minimization (SRM) (Cristianini and Shawe-Taylor 2000, Scholkopf and Smola 2001).

$y=f(x)=W^{T} x+b=\sum_{i=1}^{N} W_{i} x_{i}+b$

where $W$ represents an $\mathrm{N}$-dimensional vector and $b$ states a scalar value. The optimal separating hyperplane constructs the maximum possible distance between the plane and the 
closest data. Using the Kuhn-Tucker condition, the optimization problem casts into the equivalent Lagrangian dual quadratic optimization problem. Therefore, the classifier is obtained based on the support vector.

The SVM was used by researchers for the regression of three different types of outcomes: binary, multinomial, and continuous. Multi-class classification is called for regression with multinomial outcomes (Cortes and Vapnik 1995, Vapnik 2013).

The SVM has no equivalent for multi-class classification for more than two classes. Therefore, the results from single two-class SVMs were just mixed (Guenther and Chih-Jen 2016). Two techniques "one versus all" and "one versus one" were widely used to create the multi-class. The "one versus one" was applied by the Stata implementation: If $n$ stands for the number of classes, the support vector classification method is performed $n(n-1) / 2$ times for each pair of classes. For each pair, a point is received by the class, and the winner is the class that collects the most points of all two-class. The "one versus one" approach is outstandingly more accurate for practical applications (Chih-Wei and Chih-Jen 2002), and the training time for "one versus all" is longer than that of "one versus one". The syntax has no change during the run of multi-class classification. SVMs will automatically detect a multiclass situation and invoke one against one. Therefore, "the one-against-one" strategy is used for classifying faults in this study.

\section{Proposed Intelligent Method}

In this section, a novel intelligent multi-FD method for rolling bearings is addressed. The proposed method is a combination of the SWD, the FE, the OCDET, the PSOSCALF, and the SVM. In this method, some statistical features in the time-frequency domain are extracted consequence of decomposing vibration signals by the SWD. Then, the optimal feature set is selected using the presented OCDET. Finally, the most informative features are utilized as the input values of the optimized SVM. The flowchart of the proposed method is shown in Fig. 3.

The presented IFD technique is explained as follows:

1. 70 percentages of the vibration signals are considered as "training datasets for parameter estimation" and the remains are assigned for "final test datasets".

2. Each vibration signal of "training datasets for parameter estimation" is decomposed by the SWD. 
3. For each OCs extracted by the SWD, 42 statistical features are provided by computing the statistical functions in the time domain. Finally, the feature matrix (FM) is constructed. In this matrix, the rows and columns are corresponding to the vibration signals and features, respectively.

4. For each column of the FM, the data are normalized into the interval $[0,1]$.

5. The normalized data from the previous step are separated into "training datasets" and "validation datasets". The training and validation datasets are used to make and train SVM, respectively.

6. The PSOSCALF technique is utilized to optimize the SVM parameters ( $C$ and $\sigma$ ) and simultaneously the parameter $\xi$ in the CDET method. For this purpose, the objective function is introduced as follows (Zhang et al. 2018b):

$$
\text { objective function }=\left((\alpha-1) \frac{\text { Incorrectly recognized }}{\text { Total samples }}+\alpha \frac{R}{N} \times 100\right.
$$

where $R$ and $\mathrm{N}$ represent the number of the selected features in each stage and the number of overall features, respectively. Zhang et al. (2018b) proposed 0.01 for the parameter $\alpha$ which is adopted in this study. The upper and lower band of variables are shown in Table 2.

7. To assess the performance of the suggested method, the final test datasets are used. For this purpose, using the optimized SVM in step 6, the feature class, as well as the condition of bearing, is distinguished. 


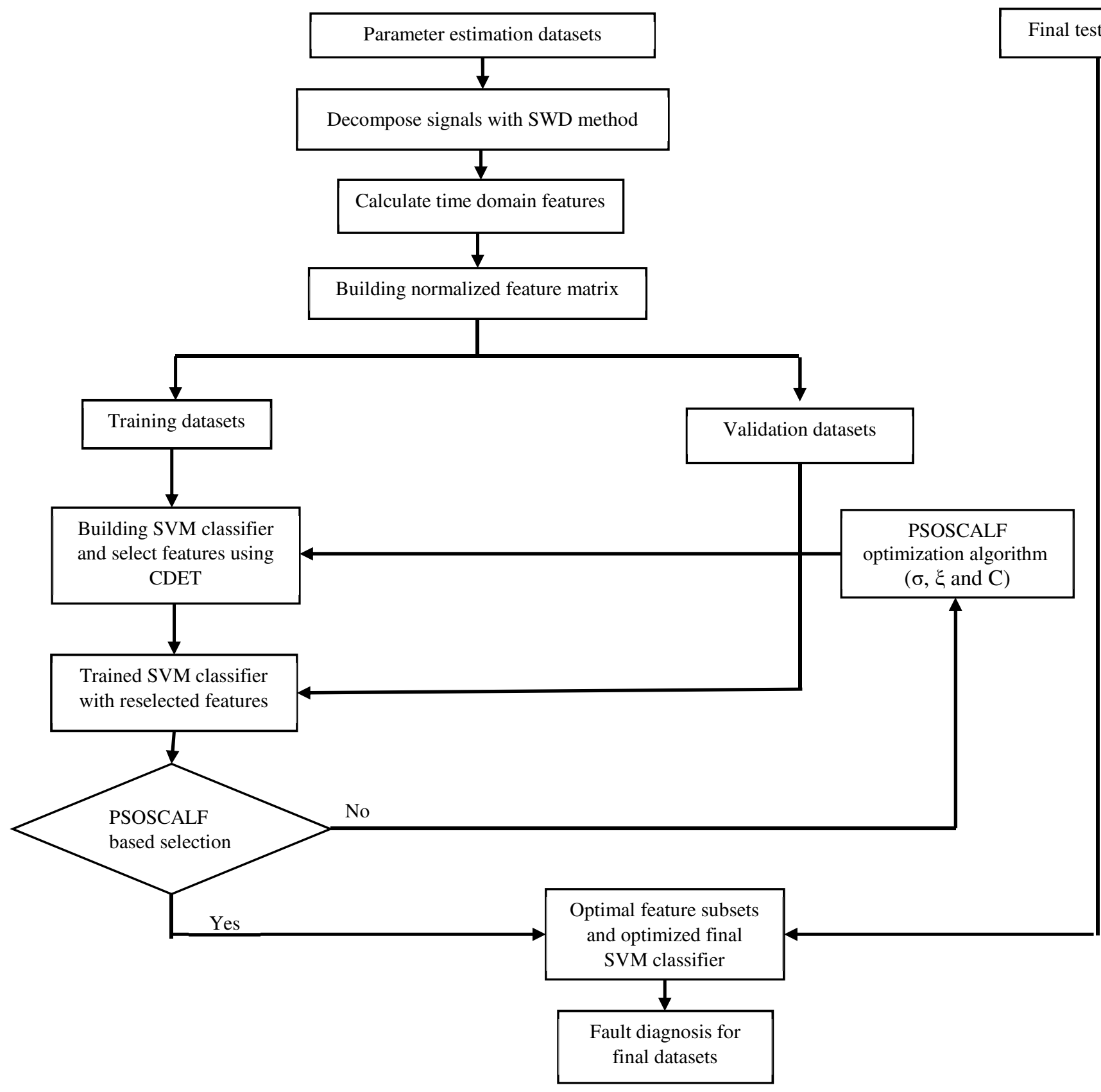

Fig. 3 The flowchart of the proposed IFD method.

Table 2 The bands of the design variables.

\begin{tabular}{lll}
\hline Parameter & Lower band & Upper band \\
\hline$C$ & $e^{-5}$ & $e^{5}$ \\
\hline$\sigma$ & $e^{-5}$ & $e^{5}$ \\
\hline$\xi$ & 0 & 1 \\
\hline
\end{tabular}




\section{Simulated Validation}

To show the effectiveness of the SWD technique, a simulated multicomponent signal corresponding to the defective bearing is employed. The complicated signal is constructed based on the mathematical model defined as follows (Nezamivand Chegini 2019a):

$$
\begin{aligned}
& X(t)=X_{1}(t)+n(t) \\
& X_{1}(t)=\sum_{i=1}^{3} A_{i} e^{-\alpha_{i}} \sin \left(2 \pi f_{i} t\right)+0.2 \sin \left(2 \pi f_{r}\right)+0.1 \sin \left(2 \pi\left(2 f_{r}\right) t\right) \\
& n(t)=N(0,0.65) \\
& t^{\prime}=\bmod \left(t, \frac{1}{f_{c h}}\right)
\end{aligned}
$$

where $X(t), X_{1}(t)$ and $n(t)$ represent a simulated signal with added noise, the noise-free signal, and the white noise with the Gaussian distribution, respectively. In the M-file environment of MATLAB ${ }^{\circledR}$, “mode function" is applied to evaluate the modulus after division. The parameters $\alpha_{i}$ are considered 100, 500, and 300, respectively. $f_{i}$ is the resonant frequency and its values are set to 500, 2500, and 4000 in Hz. $f_{r}$ is the shaft rotation frequency and its value is considered as $20 \mathrm{~Hz} .2 f_{r}$ represents the first harmonic of the shaft rotational frequency and is set to $40 \mathrm{~Hz}$. $f_{c h}$ denotes the characteristics frequency of the defect of rolling bearing. The simulated signal contains 4000 samples while the value of $12500 \mathrm{~Hz}$ is considered for sampling frequency. In Fig. 4, the noise-free signal $\left(X_{1}(t)\right)$, the added noise $(n(t))$, the simulated signal or noisy signal $(X(t))$, and the ES of the noisy signal are demonstrated. As seen in Fig. 4 (c), impulses caused by defects are hardly distinguished in the noisy signal $X(t)$.

The simulated signal with added noise has been analyzed by the SWD, EMD, CEEMDAN, and VMD techniques. The IMFs obtained by the EMD and CEEMDAN techniques are plotted in Figs. 5 and 6, respectively. The ESs of the IMFs shown in Figs. 5 and 6 are represented in Figs. 7 and 8, respectively. As can be seen in Figs. 7 and 8, among the IMFs obtained by the EMD and CEEMDAN techniques, the characteristic frequency and its harmonics appear only in the ES of the first IMF. In other words, most IMFs are not useful for fault detection. The same simulated signal is also analyzed by the VMD approach. If the mode number is chosen as 3 and 4, the decomposition results are illustrated in Figs. 9 and 10, respectively. Examination of the modes and their spectra indicates that the fault impulses emerge in only one of the modes extracted by VMD, and other modes are not suitable for 
detecting faults. The OCs extracted by the SWD method from the simulated noisy signal $(X(t))$ and their ESs are depicted in Fig. 11. As shown in Fig. 11, appearing the characteristics frequency, $f_{c h}$, and its harmonics for all OCs are distinguished and illustrated the presence of the bearing defect, clearly. The above analysis proves that the SWD approach has a better performance than other signal processing methods such as EMD, CEEMDAN, and VMD in extracting fault-related information.

(a) Signal

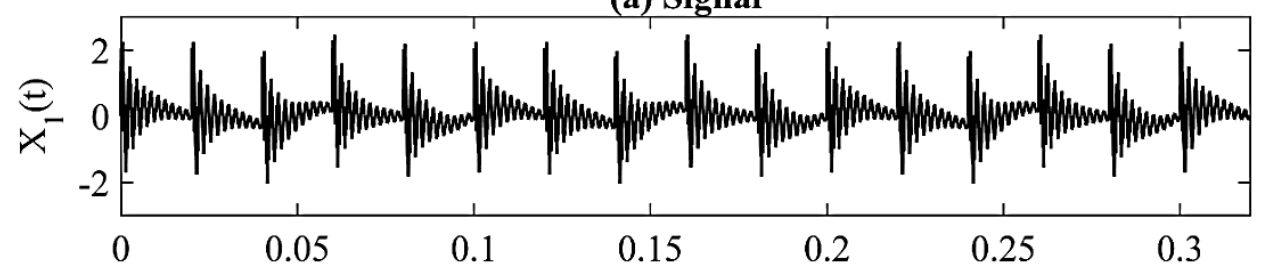

(b) Noise

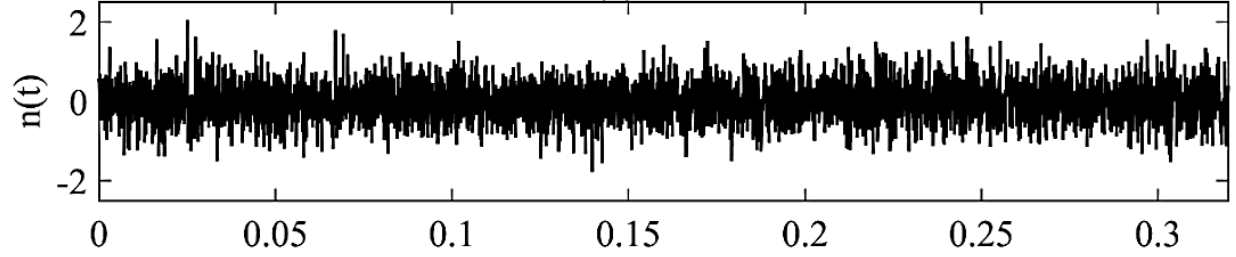

(c) Noisy Signal

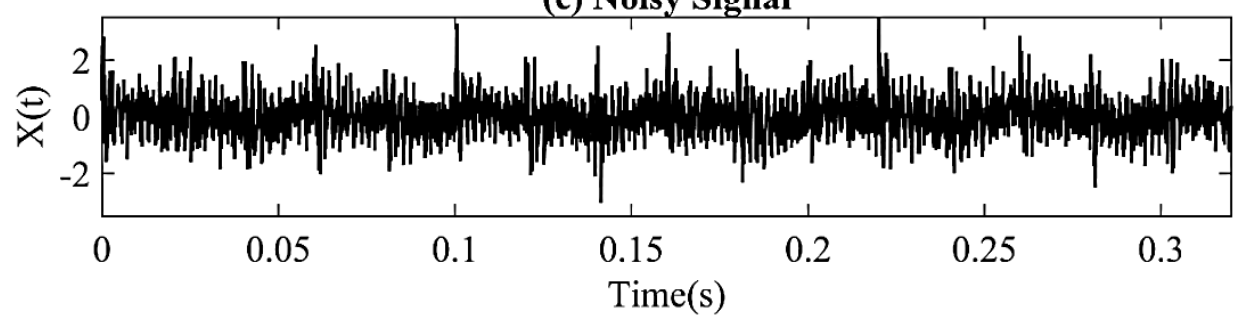

(d) Envelope spectrum of noisy signal

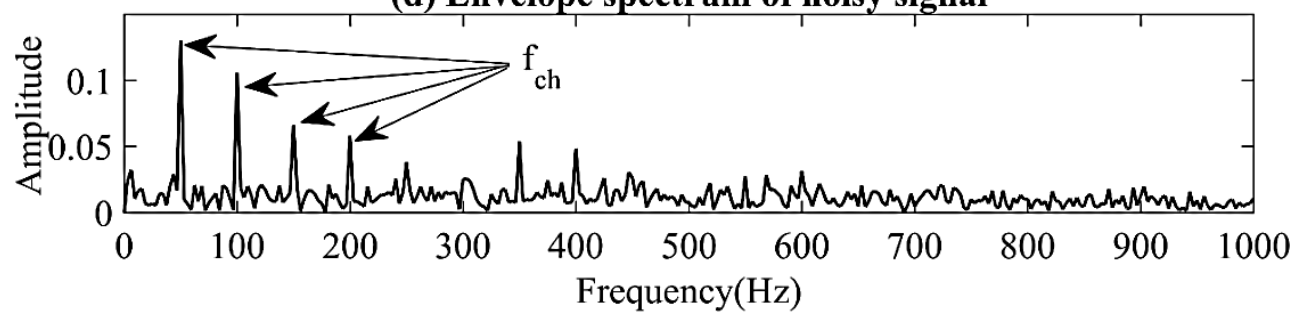

Fig. 4 a) the noise-free signal, b) added noise, c) the noisy simulated signal, d) the ES of the noisy signal. 

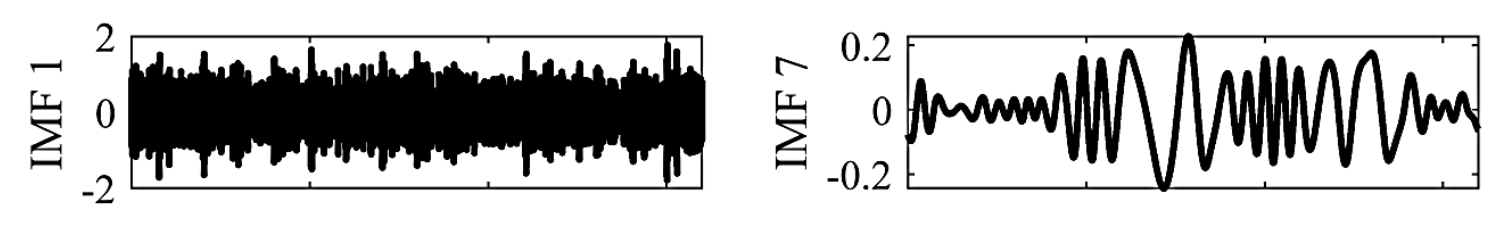

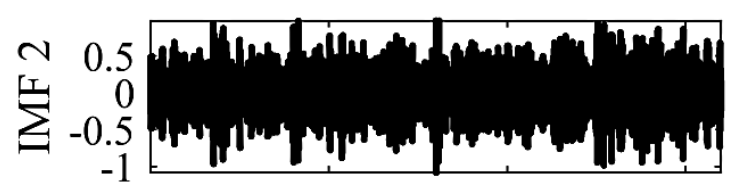

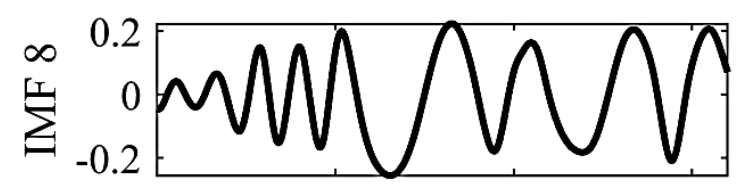

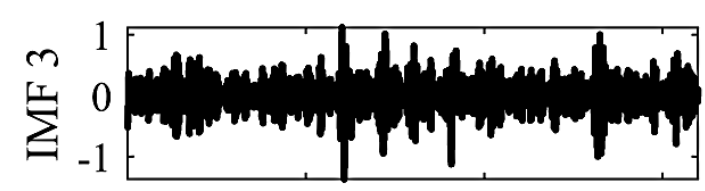

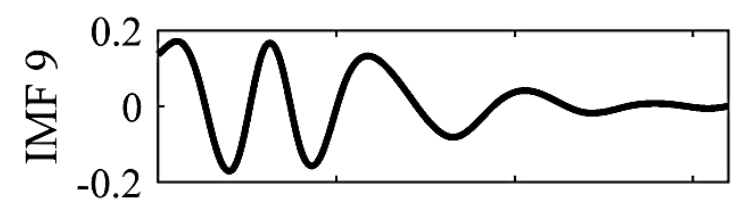

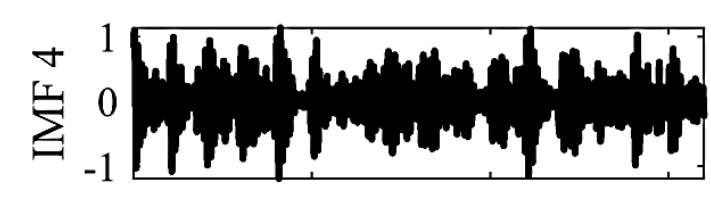
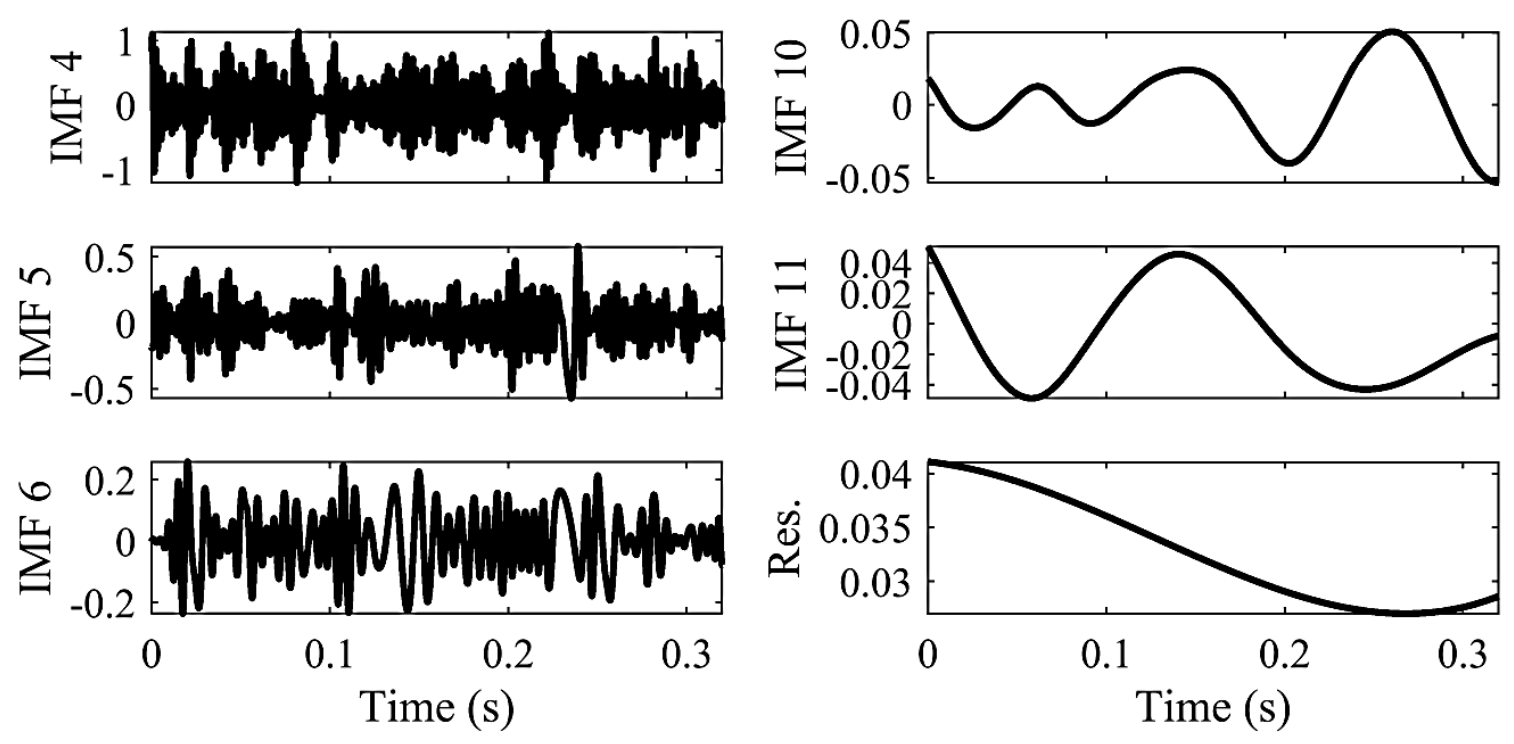

Fig. 5 The IMFs of the noisy signal obtained by EMD. 

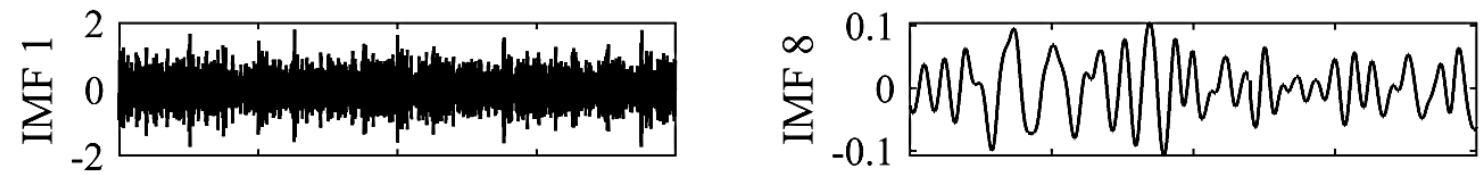

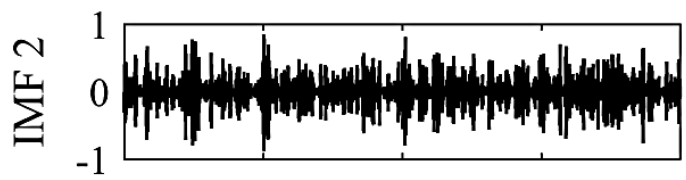

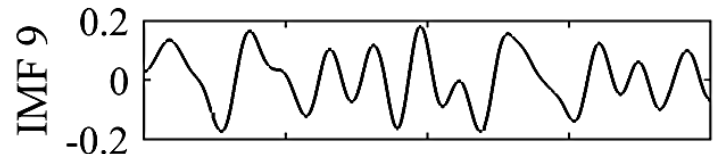

$\sum_{-0.5}^{m} \begin{array}{rr}0.5 \\ \sum_{-1}\end{array}$

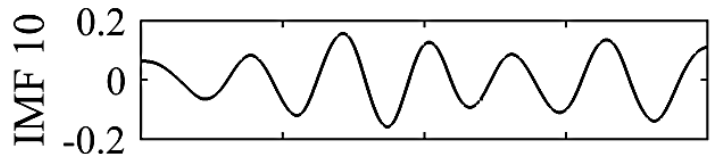

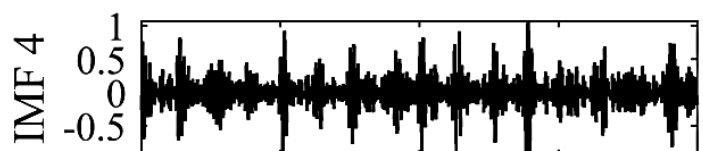
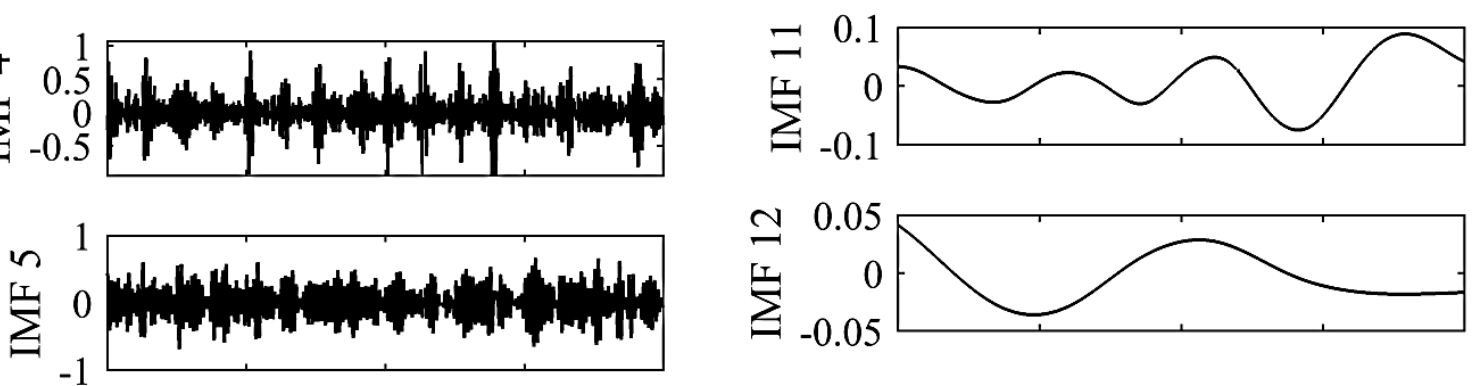

$\sum_{-0.5}^{0} \begin{array}{ll}0.5 \\ n\end{array}$
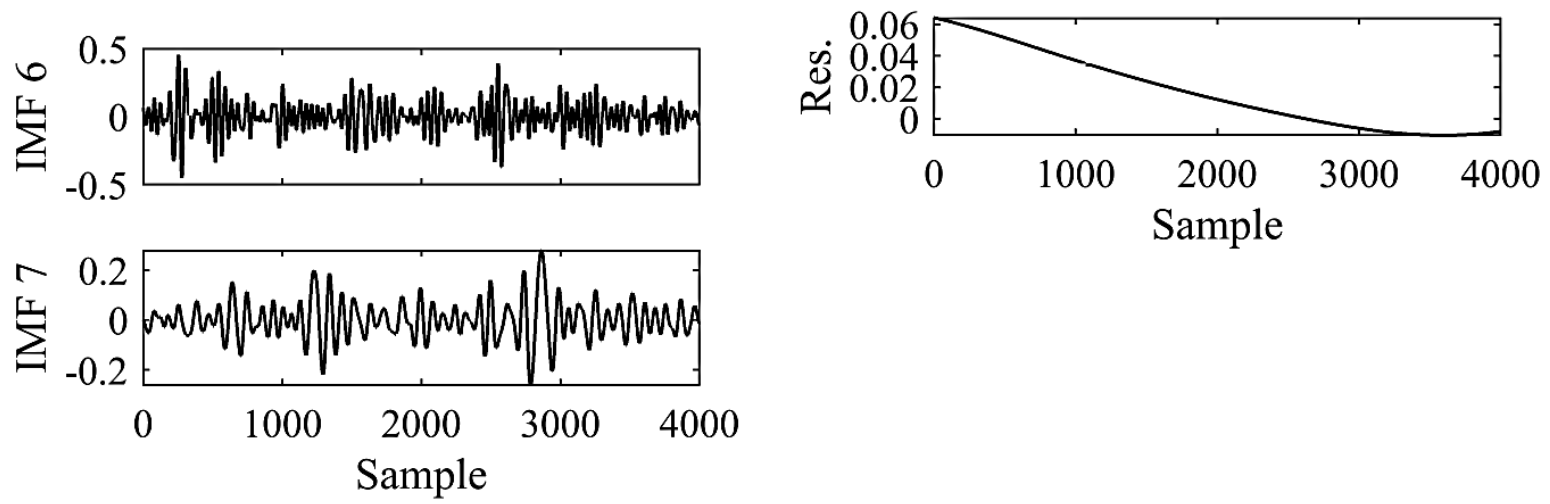

Fig. 6 The IMFs of the noisy signal obtained by CEEMDAN. 

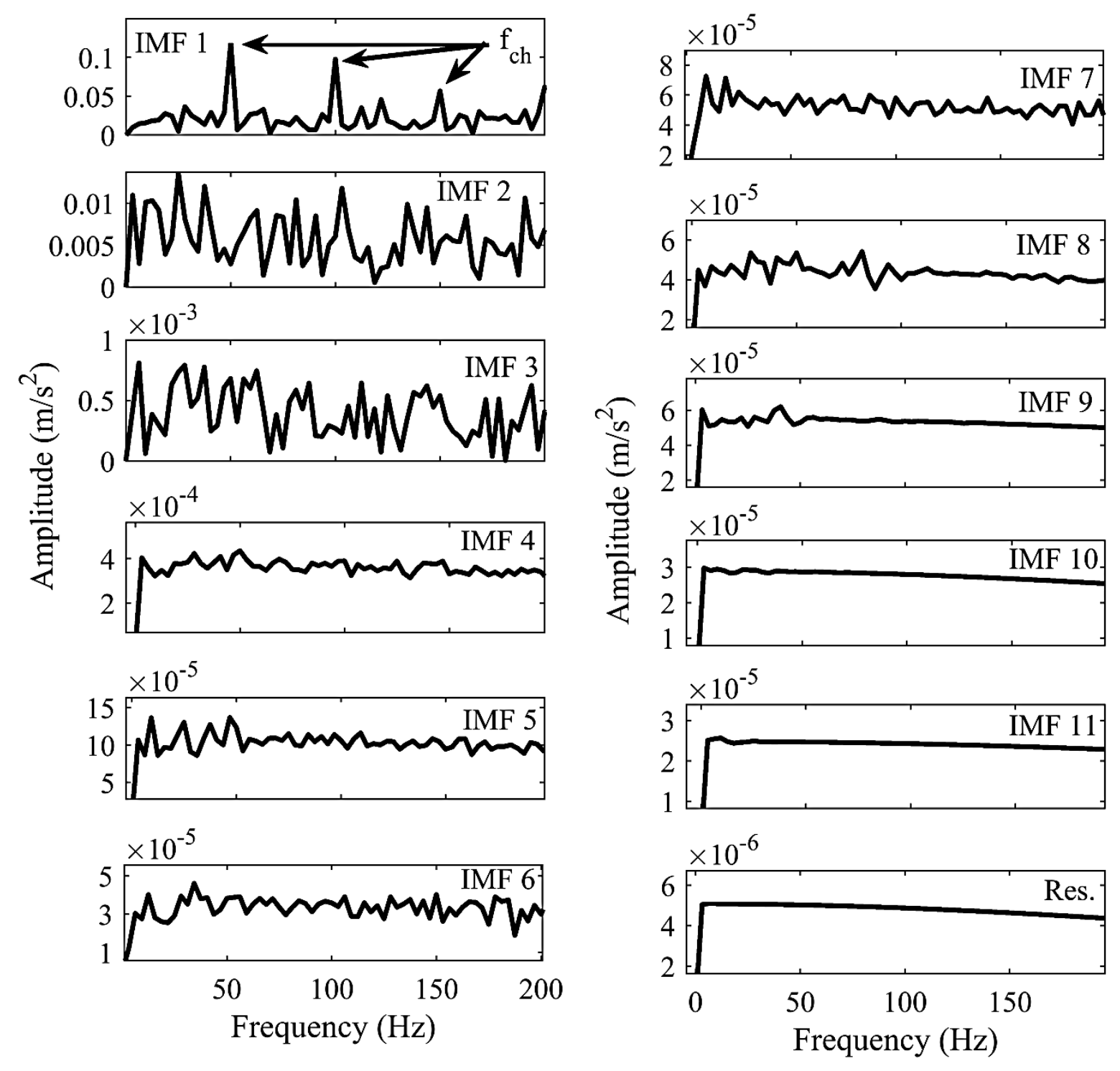

Fig. 7 The ESs of the IMFs obtained by EMD. 

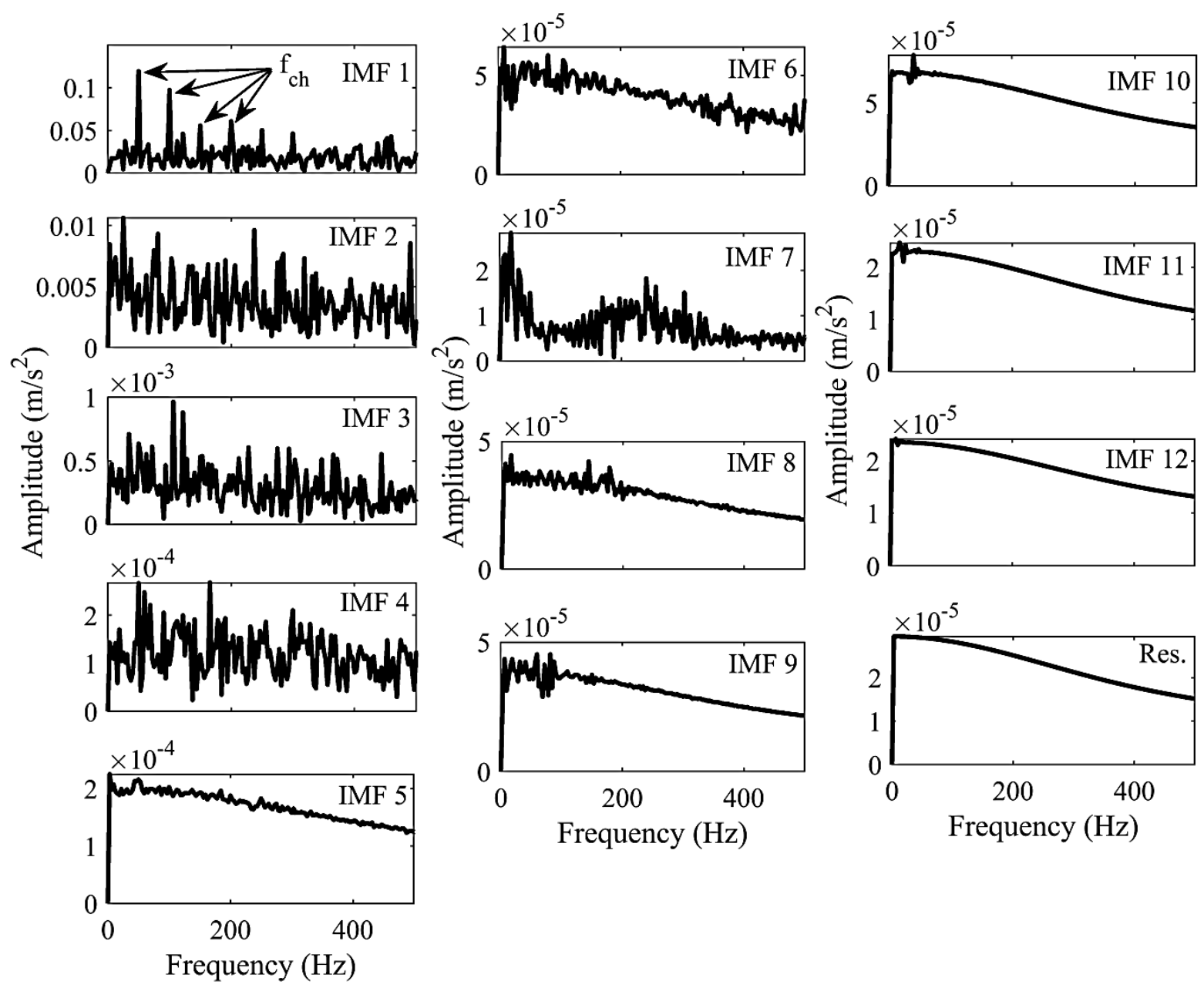

Fig. 8 The ESs of the IMFs obtained by CEEMDAN. 

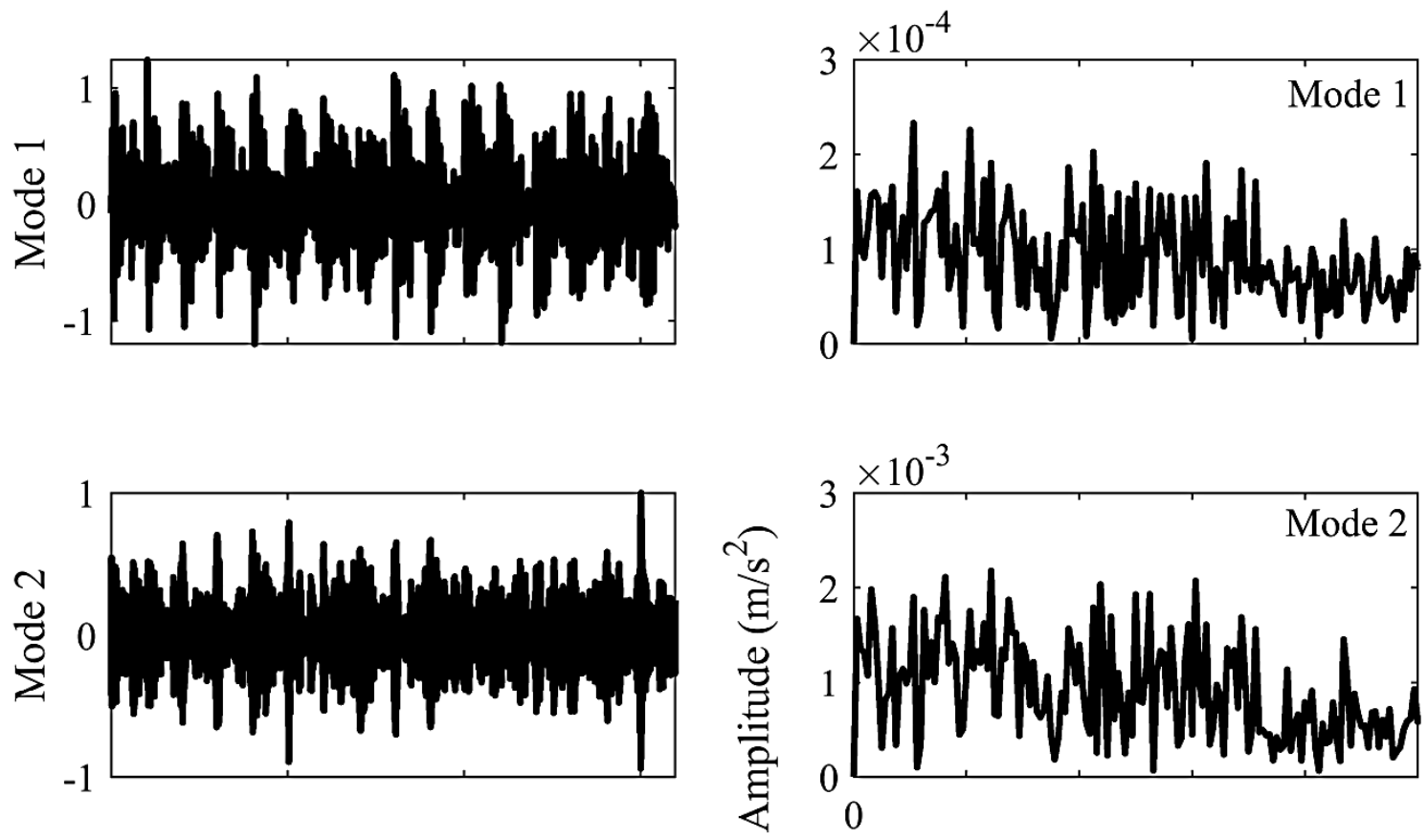

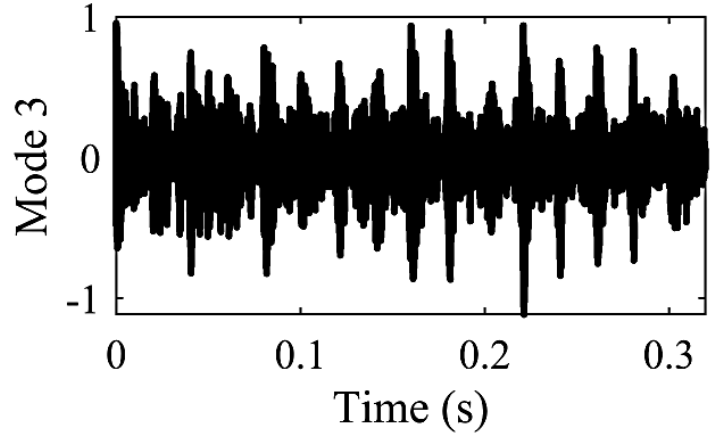

(a)

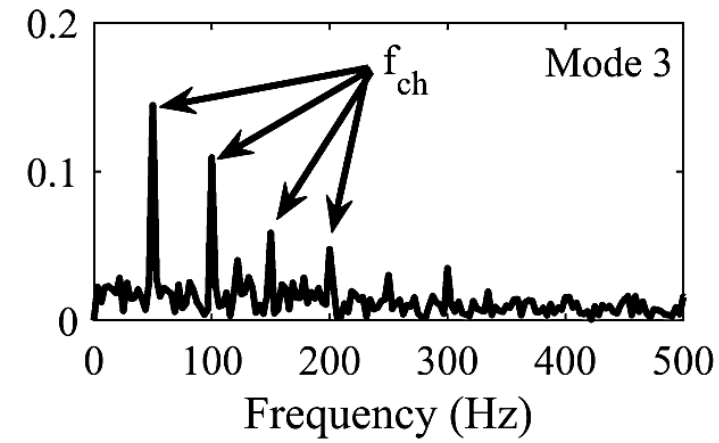

(b)

Fig. 9 The decomposition results of VMD when the mode number is 3. a) the modes, b) the ESs of the modes. 

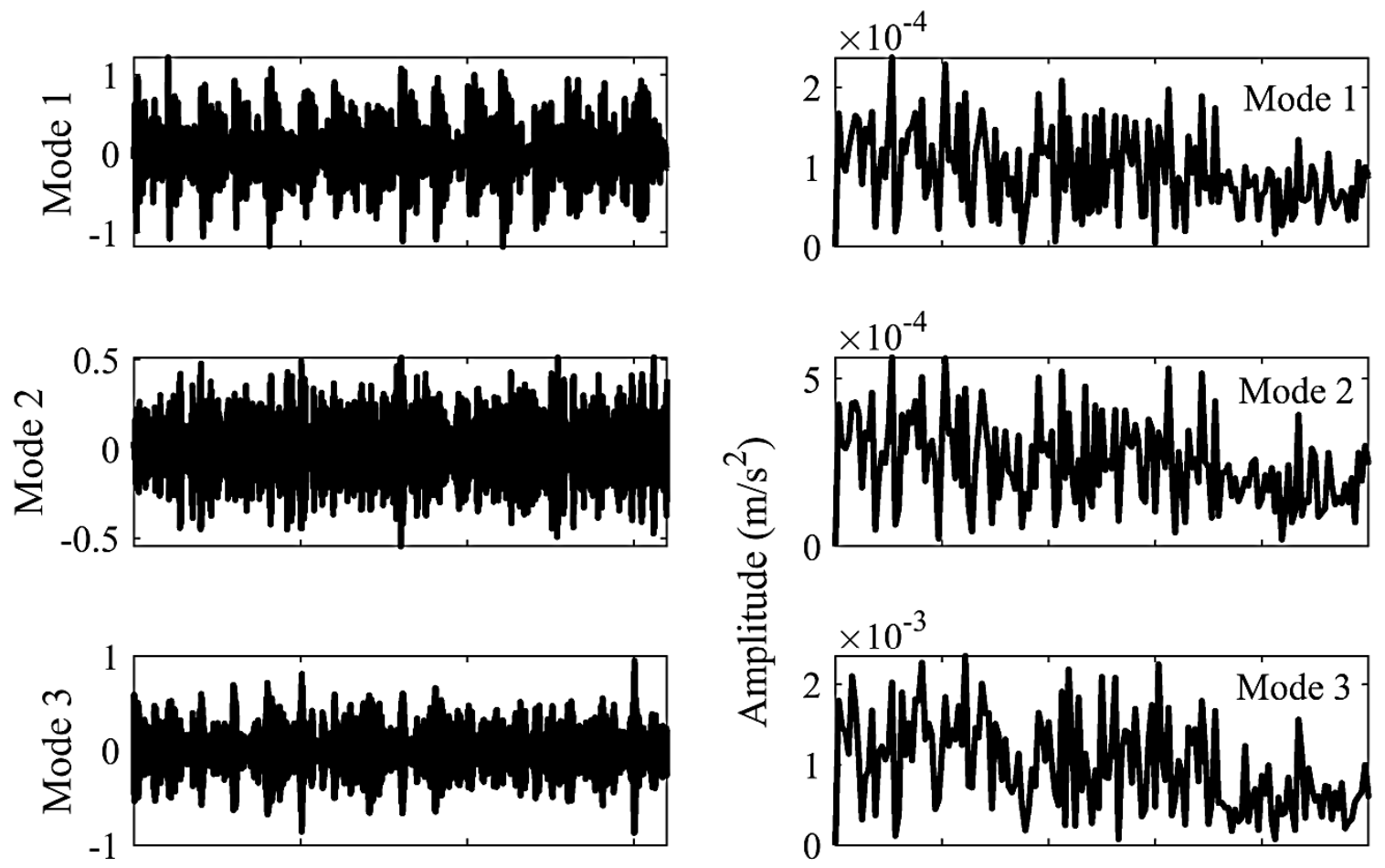

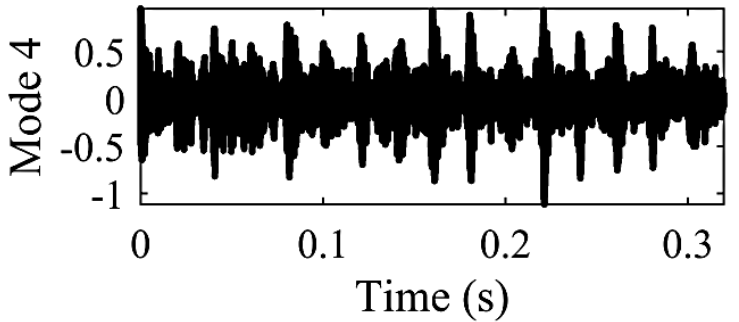

(a)

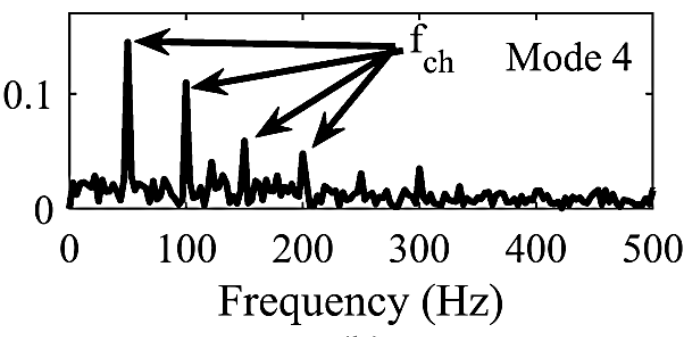

(b)

Fig. 10 The decomposition results of VMD when the mode number is 4. a) the modes, b) the ESs of the modes. 

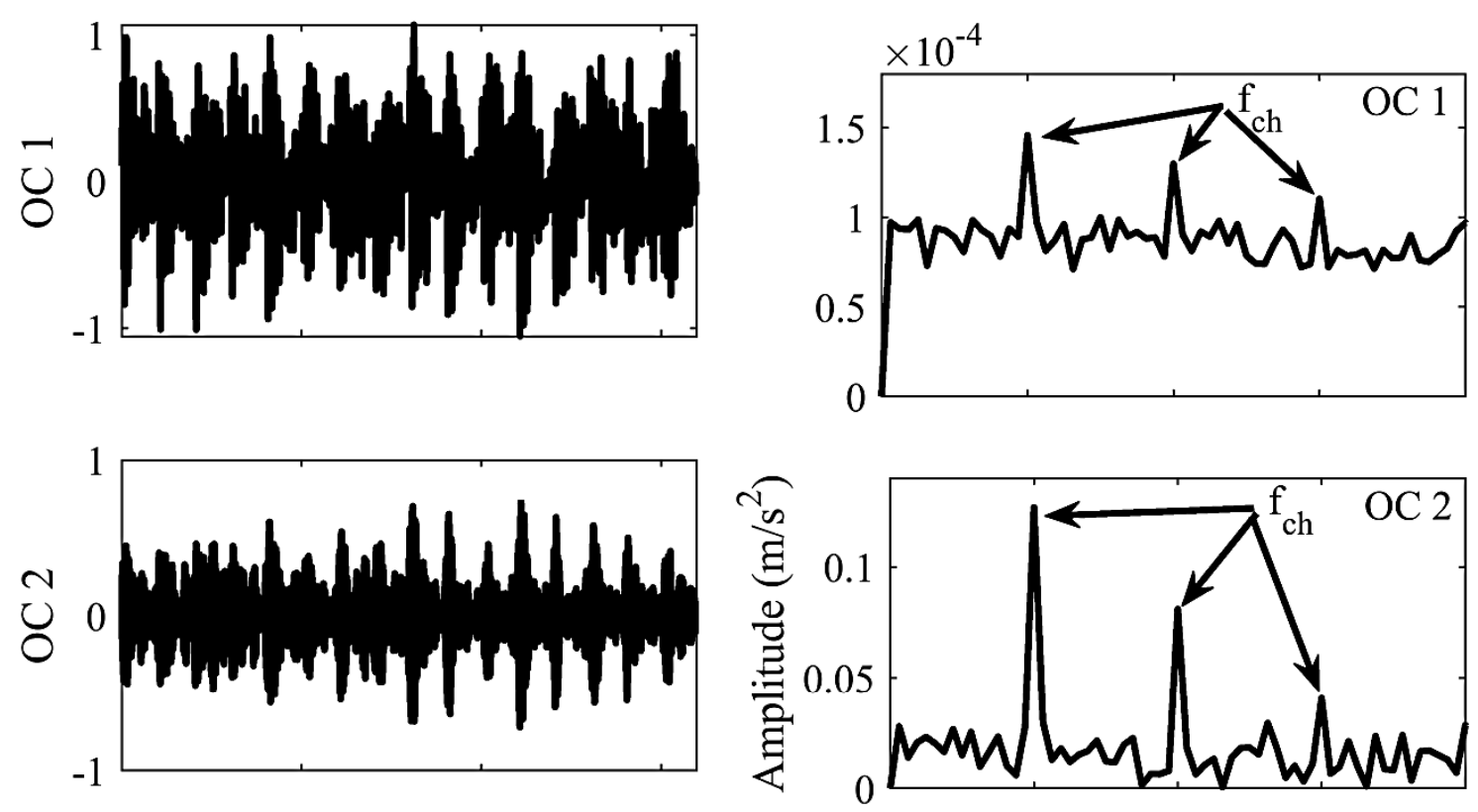

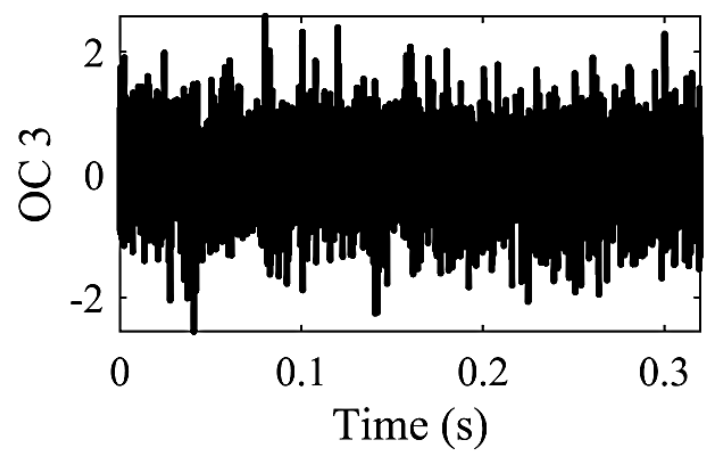

(a)

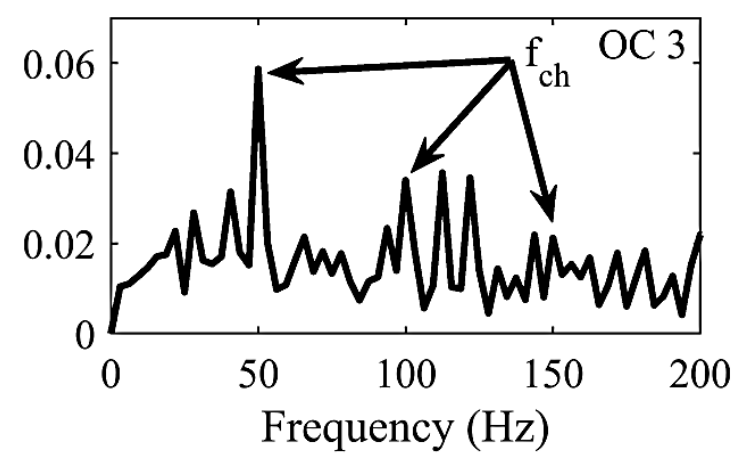

(b)

Fig. 11 The results obtained by the SWD method for the simulated signal. a) the OCs, b) the ESs of the OCs.

\section{Validation Datasets}

\subsection{The Case Western Reverse University Bearing Data Set}

To illustrate the effectiveness of the proposed intelligent method, the vibration signals of bearing have been adopted from the Bearing Data Centre of Case Western Reverse University (CWRU) are used (Bearing Data Centre 2016). The setup consists of a motor, a coupling, and a dynamotor as shown in Fig. 12. The bearing type of SKF 6205-2RS JEM was used to obtain the vibration signals. Table 3 presents the geometrical characteristic of this bearing. By using electro-discharge machining, defects were produced in the bearings. It is worth mentioning that the defects consist of 4 different diameters $(0.1778 \mathrm{~mm}, .03556 \mathrm{~mm}$, $0.5334 \mathrm{~mm}$, and $0.7112 \mathrm{~mm}$ ) with a constant depth of $0.2794 \mathrm{~mm}$. These faults were created 
at the outer race, inner race, and rolling element of the bearings. The vibration data were measured by the Kistler accelerometer which was placed at the top of the drive end with a sampling frequency of $12000 \mathrm{~Hz}$. The vibration signals were collected at 4 different rotating speeds of 1797, 1772, 1750, and $1730 \mathrm{rpm}$ and 4 different loads of $0,1,2$, and $3 \mathrm{hp}$.

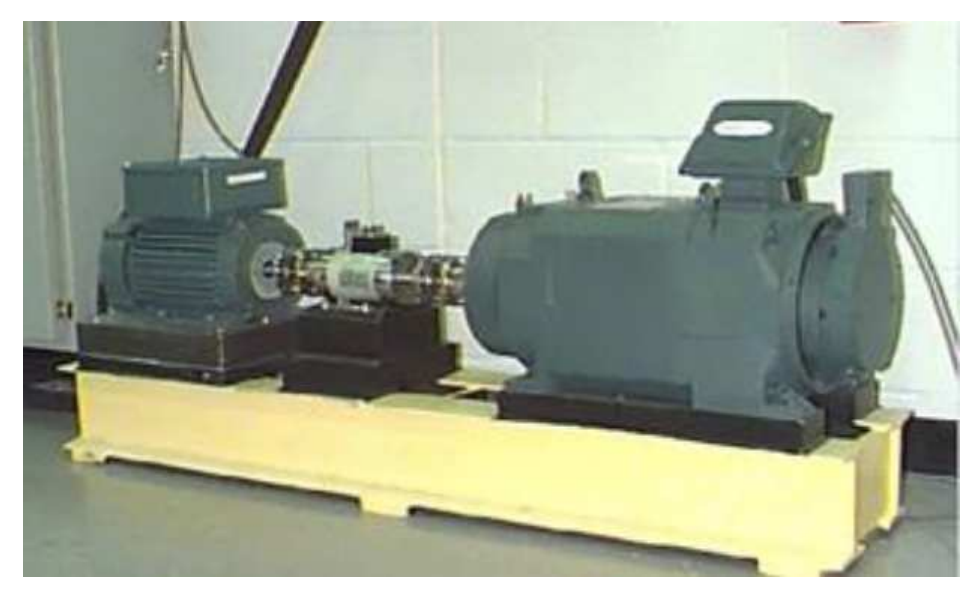

(a)

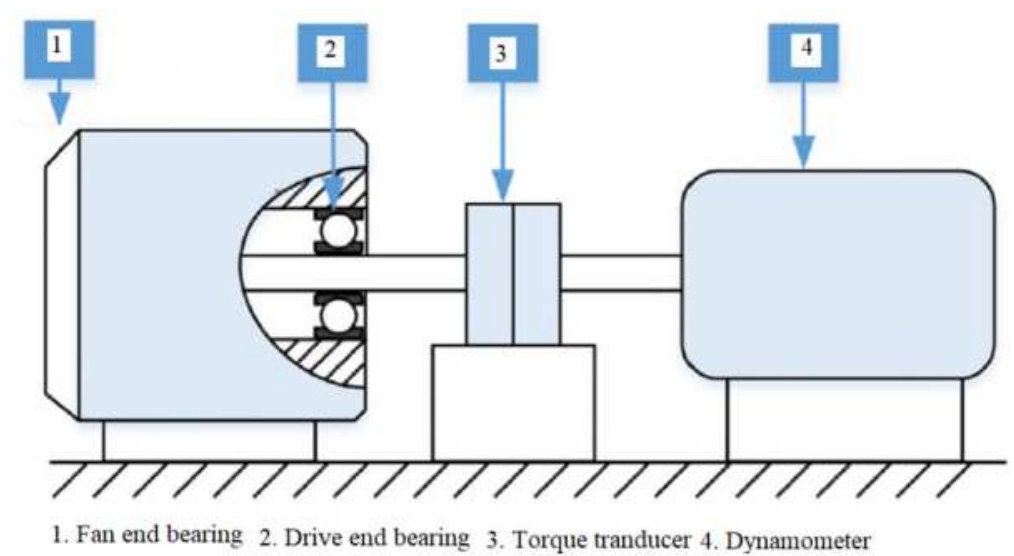

(b)

Fig. 12 a) Experimental set, b) The schematic of the experimental set (Bearing Data Center 2016).

Table 3 The detailed parameters of the ball bearing 6205-2RS JEM SKF (Bearing Data Center 2016).

\begin{tabular}{l|l}
\hline Parameter & Value(millimetres) \\
\hline Inside Diameter & 25 \\
\hline Outside Diameter & 51 \\
\hline Thickness & 15 \\
\hline Ball Diameter & 0.7940 \\
\hline Pitch Diameter & 3.9039 \\
\hline
\end{tabular}

Table 4 lists the properties of vibration signals as well as the number of cases study used in this work. Case 1 was used to examine the ability of the suggested method in the early 
detection of the faulty type at different speeds with the smallest fault diameter $(0.1778 \mathrm{~mm})$. Case studies 2-4 were used to detect the different fault sizes at various rotational speeds. As an example, the vibration signals with defect size $0.1778 \mathrm{~mm}$ at speed $1797 \mathrm{rpm}$ are illustrated in Fig. 13. According to Fig. 13, it is evident that the properties of the vibration signals $e . g$., shocks, and amplitudes depend on the type of defects. However, it is difficult to diagnose the defect type and its severity by merely evaluating these characteristics. Hence, it seems necessary to develop a powerful intelligent method for extracting the features and diagnosing the fault characteristics.

Table 4 Description of the case studies used in the presented method.

\begin{tabular}{|c|c|c|c|c|c|}
\hline Case studies & $\begin{array}{l}\text { Number } \\
\text { of class }\end{array}$ & $\begin{array}{l}\text { Number of } \\
\text { training } \\
\text { samples }\end{array}$ & $\begin{array}{l}\text { Number of } \\
\text { testing } \\
\text { samples }\end{array}$ & $\begin{array}{l}\text { Working } \\
\text { condition }\end{array}$ & Fault size $(\mathrm{mm})$ \\
\hline Case 1: fault identification & 4 & 56 & 24 & $\begin{array}{l}\text { Normal } \\
\text { Inner race, outer } \\
\text { race and rolling } \\
\text { element }\end{array}$ & -- \\
\hline $\begin{array}{l}\text { Case 2: fault level } \\
\text { identification for inner race }\end{array}$ & 5 & 52 & 23 & $\begin{array}{l}\text { Normal } \\
\text { Inner race }\end{array}$ & $\begin{array}{l}-- \\
0.1778 \sim 0.7112\end{array}$ \\
\hline $\begin{array}{l}\text { Case 3: fault level } \\
\text { identification for outer race }\end{array}$ & 4 & 56 & 24 & $\begin{array}{l}\text { Normal } \\
\text { Outer race }\end{array}$ & $\begin{array}{l}--- \\
0.1778 \sim 0.5334\end{array}$ \\
\hline $\begin{array}{l}\text { Case 4: fault level } \\
\text { identification for rolling } \\
\text { element }\end{array}$ & 5 & 70 & 30 & $\begin{array}{l}\text { Normal } \\
\text { Rolling element }\end{array}$ & $\begin{array}{l}--- \\
0.1778 \sim 0.7112\end{array}$ \\
\hline
\end{tabular}



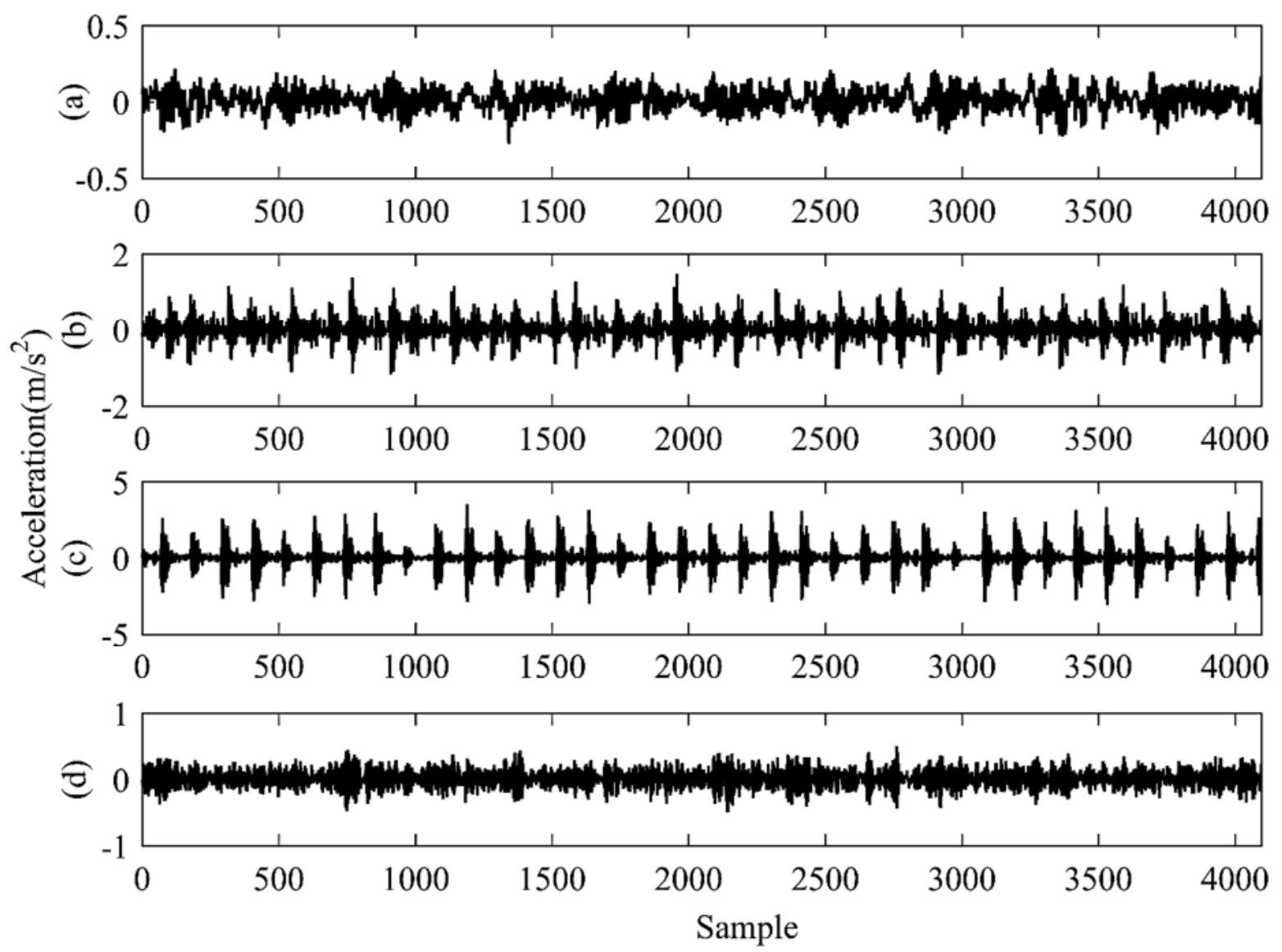

Fig. 13 The vibration signals at speed of $1797 \mathrm{rpm}$ for four different cases $(0.1778 \mathrm{~mm})$. a) normal, b) inner race, $c$ ) outer race, d) rolling element

\subsubsection{Optimal Feature Set}

When a fault occurs in one of the bearing components, intermittent impulses appear in the vibration signal. The frequency of the impulses plays an important role in determining the location of the defect in the bearing components. In other words, each type of fault corresponds to a frequency called the bearing characteristic frequency. With the emergence of each of the characteristic frequencies and its harmonics in the vibration signal spectrum, the location of the defect in the bearing can be distinguished. In (Nezamivand Chegini et al. 2019a), for the bearing used in the CWRU experimental set and the shaft rotational speed of $f_{r}=1797 \mathrm{rpm}$, the characteristic frequency of the inner ring, $f_{i}$, and the characteristic frequency of the outer ring, $f_{o}$, were reported as $162.18 \mathrm{~Hz}$ and $107.36 \mathrm{~Hz}$, respectively. The vibration signals in Figs $13 \mathrm{~b}$ and $13 \mathrm{c}$ correspond to the defective inner ring and the defective outer ring, respectively. These signals are analyzed by the SWD method. The first four OCs and their ES are illustrated in Fig. 14 and Fig. 15 for the faulty inner ring and faulty outer ring, respectively. As illustrated in Fig. 14, the shaft rotational frequency, $f_{r}$, and the characteristic 
frequency of $f_{i}$ appear in the spectrums of the first three OCs. The presence of these frequencies indicates that there is a fault in the inner ring. Fig. 15 demonstrates well the sufficiency of the SWD approach in extracting information from the vibration signal of the faulty outer ring. It can be seen in this figure that the shaft rotational frequency, $f_{r}$, and the characteristic frequency of the outer ring, $f_{o}$, and its harmonics are easily recognizable in the ESs of all OCs. The presence of these frequencies confirms the occurrence of a defect in the outer ring of the bearing. The results of Figs. 14 and 15 prove the effectiveness of the SWD technique in detecting the fault location for defects with very small sizes.

According to the flowchart depicted in Fig. 3, the number of features extracted from the vibration signals depends on the OCs obtained by the SWD method. After decomposing the signals, the statistical features in the time domain are obtained from the vibration signals. The first two OCs obtained by the SWD have been selected by the kurtosis parameter. The high dimensionality of the FM leads to increase computational complexity and also to decrease the efficiency of the SVM classifier accuracy in recognizing the bearing conditions (Zhang et al. 2018b). So, in this paper, the OCDET method is utilized to calculate the weight of each statistical feature. Consequently, the features with weights larger than of optimized threshold value have been selected. Fig.16 shows the normalized value of all statistical features computed by the OCDET. The optimal features for the four case studies described in Table 4 have been introduced in Table 5 . 

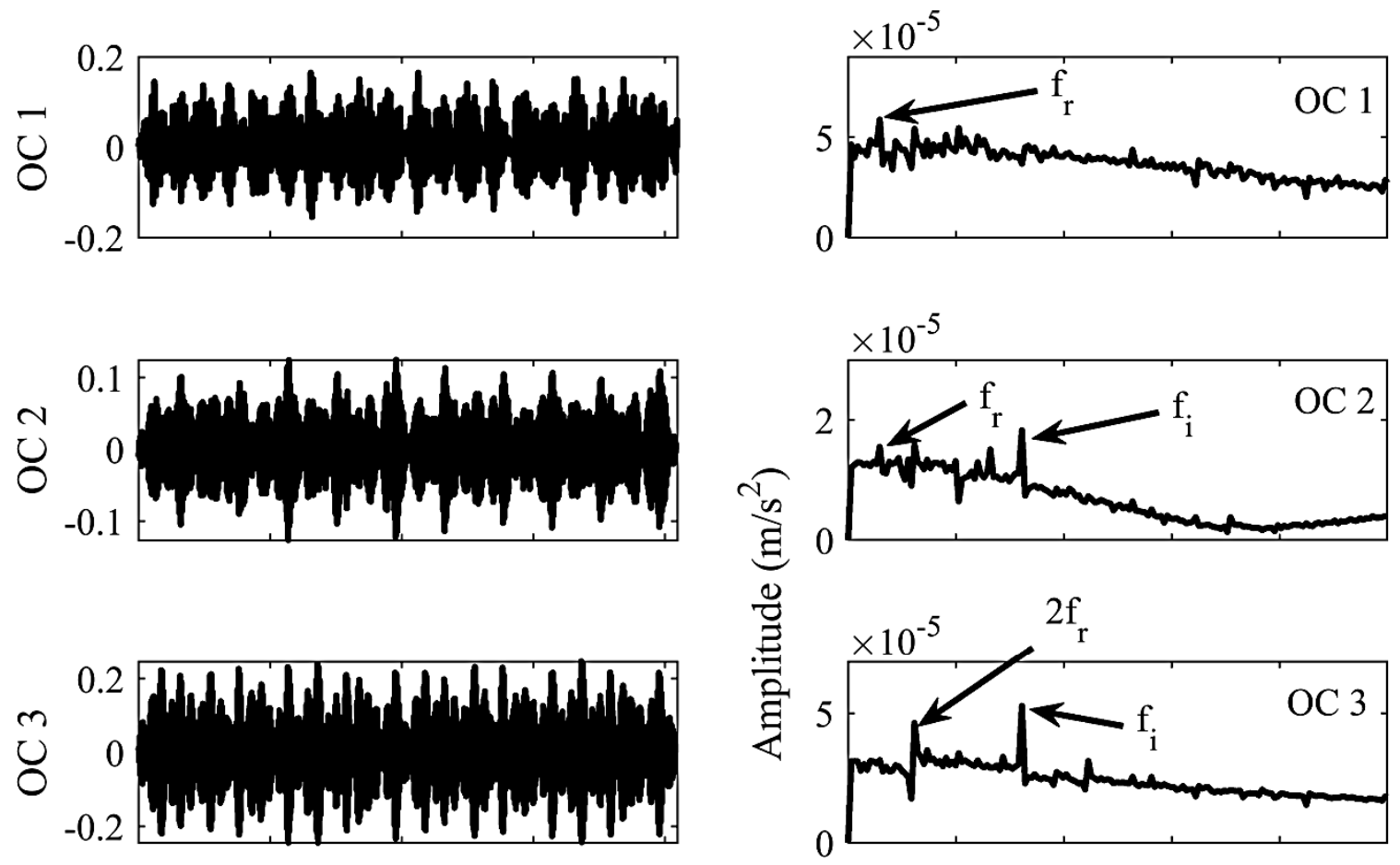

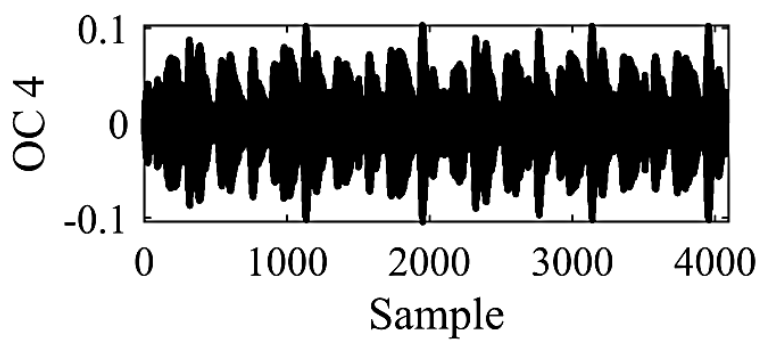

(a)

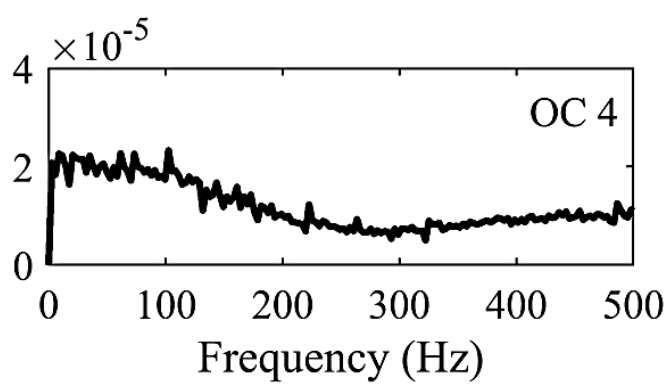

(b)

Fig. 14 The results obtained by SWD for the vibration signal of the faulty inner ring with fault depth $0.1778 \mathrm{~mm}$. a) the OCs, b) the ES of the OCs. 

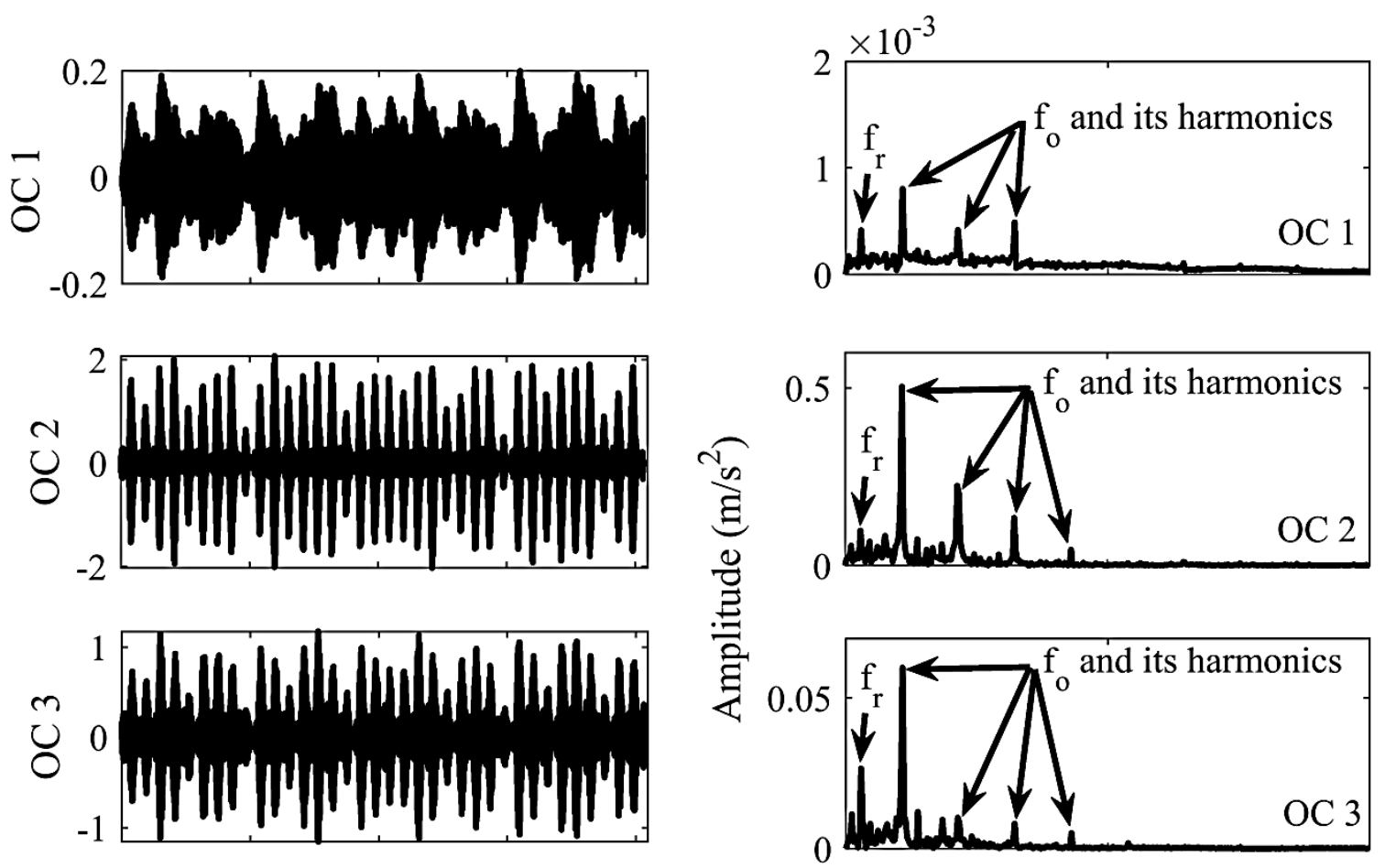

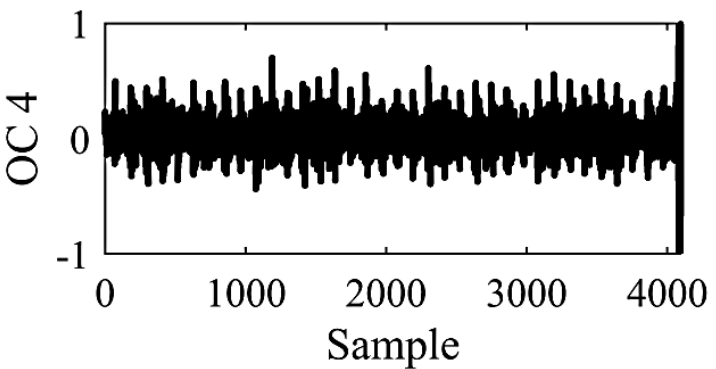

(a)

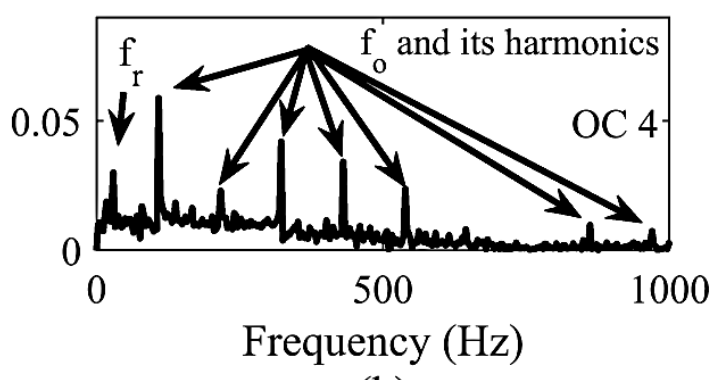

(b)

Fig. 15 The results obtained by SWD for the vibration signal of the faulty outer ring with fault depth $0.1778 \mathrm{~mm}$. a) the OCs, b) the ES of the OCs. 

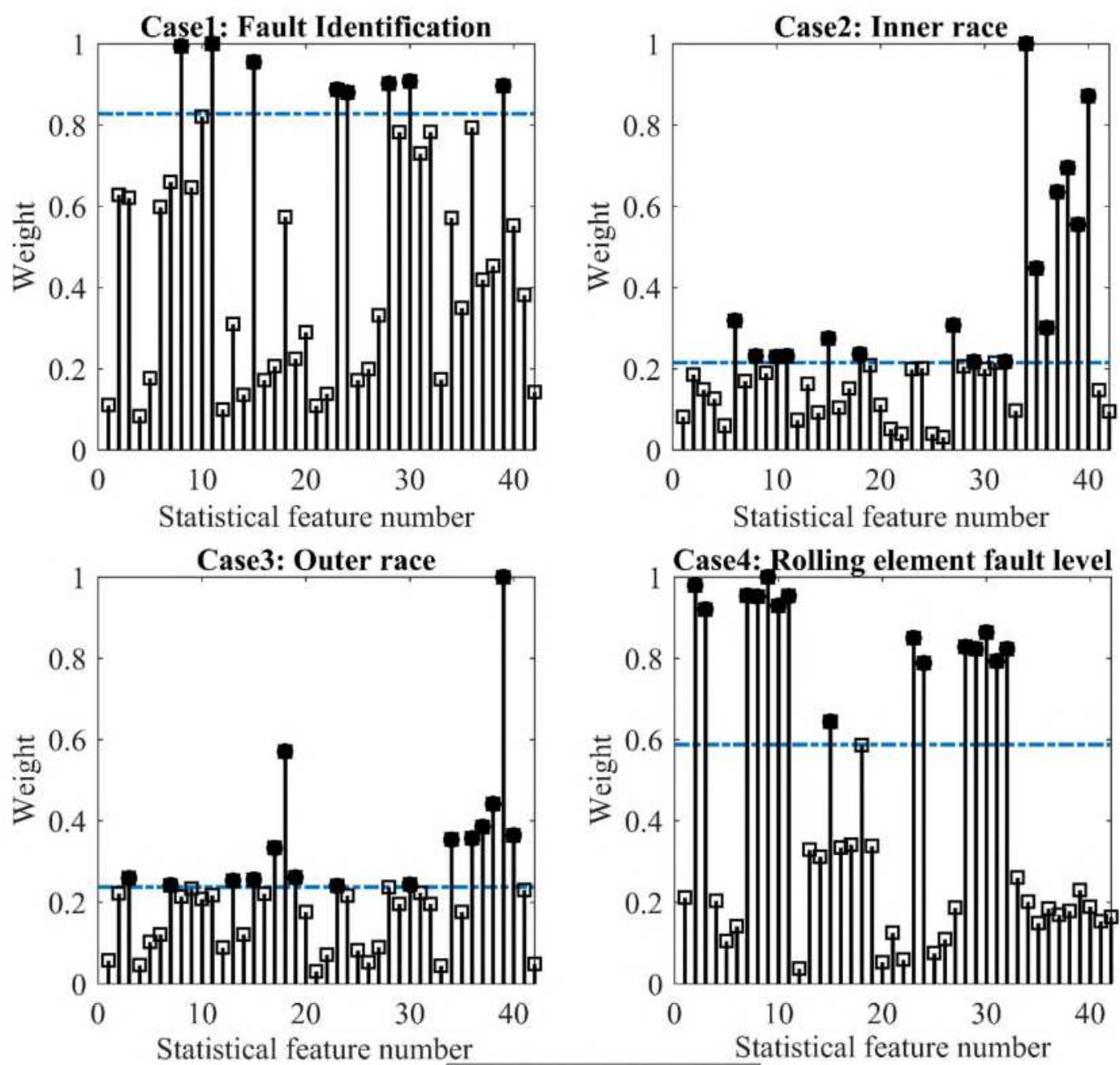

- Optimal Threshold

Statistical Feature

Optimal Feature

Fig. 16 The weight scores of all features obtained by the CDET for all case studies. 
Table 5 The best features selected by CDET for CWRU bearing data set.

\begin{tabular}{|c|c|c|}
\hline Case studies & $\begin{array}{l}\text { Optimal features for the first } \\
\text { selected OC }\end{array}$ & $\begin{array}{l}\text { Optimal features for the Second } \\
\text { selected OC }\end{array}$ \\
\hline Case 1 & $\begin{array}{l}\text { Root mean square } \\
\text { Standard deviation } \\
\text { Shape factor }\end{array}$ & $\begin{array}{l}\text { Maximum } \\
\text { Minimum } \\
\text { Range } \\
\text { Peak value } \\
\text { Hybrid feature 1 }\end{array}$ \\
\hline Case 2 & $\begin{array}{l}\text { Entropy } \\
\text { Root mean square } \\
\text { Mean peak } \\
\text { Standard deviation } \\
\text { Shape factor } \\
\text { Hybrid feature } 1\end{array}$ & $\begin{array}{l}\text { Entropy } \\
\text { Root mean square } \\
\text { Standard deviation } \\
\text { Kurtosis } \\
\text { Crest factor } \\
\text { Shape factor } \\
\text { Impulse factor } \\
\text { Clearance factor } \\
\text { Hybrid feature } 1 \\
\text { Hybrid feature } 2\end{array}$ \\
\hline Case 3 & $\begin{array}{l}\text { Minimum } \\
\text { Range } \\
\text { Kurtosis } \\
\text { Shape factor } \\
\text { Clearance factor } \\
\text { Hybrid feature 1 } \\
\text { Hybrid feature 2 }\end{array}$ & $\begin{array}{l}\text { Maximum } \\
\text { Peak value } \\
\text { Kurtosis } \\
\text { Shape factor } \\
\text { Impulse factor } \\
\text { Clearance factor } \\
\text { Hybrid feature 1 } \\
\text { Hybrid feature 2 }\end{array}$ \\
\hline Case 4 & $\begin{array}{l}\text { Maximum } \\
\text { Minimum } \\
\text { Range, Root mean square } \\
\text { Peak value } \\
\text { Mean peak } \\
\text { Standard deviation } \\
\text { Shape factor }\end{array}$ & $\begin{array}{l}\text { Maximum } \\
\text { Minimum } \\
\text { Range } \\
\text { Root mean square } \\
\text { Peak value } \\
\text { Mean peak } \\
\text { Standard deviation }\end{array}$ \\
\hline
\end{tabular}

\subsubsection{Comparison with Other Techniques}

In this section, the obtained results of the proposed technique are compared with that of other methods explained in Table 6. Method 1 (Yin et al. 2014) applies the BPSO algorithm to select the most appropriate features from the high dimensional feature set and to optimize the SVM parameters. Method 1 is selected to investigate the consequence of applying the OCDET technique in the proposed scheme of this study. In Method 2 (Zhang et al. 2018b), the vibration data are decomposed utilizing the ITD method. The features are ranked by the Relief-F algorithm and then, the pre-selected features are determined. Making a comparison between the present study and Method 2 can easily validate the capability of the hybrid methods such as OCDET+SWD and Relief-F+ITD. Method 3 resembles the proposed 
method except that in Method 3, the EMD algorithm is a substitute for the SWD method. Similar to the proposed method, the SWD is also used in Method 4 for extracting the OCs, and the FDAF-score algorithm is applied to select the optimal features. On the other hand, with investigating the result of the proposed method, and Method 4, the capabilities of these techniques have been compared with each other. The steps of the FE, the FS, and the bearing condition prediction in Methods 5 and Method 6 are similar to the approach presented in this paper. However, in the optimization stage, the GMPSO algorithm (Ding et al. 2020a, b) and the GOA algorithm (Zhang et al. 2018a) are used in Methods 5 and Method 6, respectively. To evaluate the capability of the PSOSCALF algorithm in improving the FS step and the SVM classifier accuracy, the results of the proposed technique have been compared with the results of Methods 5 and Method 6.

Table 6 The description of the other FD method for comparison with the proposed method.

\begin{tabular}{ll}
\hline Method & Description \\
\hline $\begin{array}{l}\text { The proposed } \\
\text { intelligent method }\end{array}$ & $\begin{array}{l}\text { Extracting OCs by SWD +Determine the preselected features using the } \\
\text { OCDET method + Select the optimal features and compute the optimal } \\
\text { parameters of the SVM classifier using PSOSCALF algorithm+ Diagnose fault } \\
\text { by the optimal feature subsets and the optimized SVM. }\end{array}$ \\
\hline $\begin{array}{l}\text { Method 1 (Yin et } \\
\text { al. 2014) }\end{array}$ & $\begin{array}{l}\text { Choose the most sensitive features from the original feature set and use the } \\
\text { BPSO for optimizing the SVM classifier }\end{array}$ \\
\hline Method 2 (Zhang & $\begin{array}{l}\text { Apply the Relief-F method for preselecting the primary features + identify the } \\
\text { informative features and optimize the SVM using the BPSO algorithm+ }\end{array}$ \\
\hline Determine the status of the system by selected features and optimized SVM.
\end{tabular}

This method is similar to the proposed method, except that in the optimization Method 5 process, the GMPSO algorithm (Ding et al. 2020a, b) is used instead of the PSOSCALF algorithm.

Method 6

This method is similar to the proposed method, except that in the optimization process, the GOA algorithm (Zhang et al. 2018a) is used instead of the PSOSCALF algorithm.

The meta-heuristics optimization algorithms BPSO, PSOSCALF, GMPSO and GOA have been utilized in all methods explained in Table 6. Since the meta-heuristic algorithms have probabilistic nature and their solutions may be distinct for different runs, the suggested method, and other methods are run 30 times independently. Finally, the obtained results 
related to the FD accuracy such as the terms of optimal parameters, the average training accuracy, and the average testing accuracy are given in Table 7. Also, the optimal parameters of SVM and the threshold of the OCDET are reported in Table 7. On the other hand, to better compare different methods, the current work and other techniques for each case study are ranked in the training and testing stages, and the results are reported in Table 8. In this table, in each of the training and testing stages, five numerals have been reported for each method. Four numerals are related to the rank of each FD method in each case study and the fifth number is the final rank of each method in the training and testing stages. The interpretive discussion of the results reported in Tables 7 and 8 is as follows:

- According to the final rank reported in Table 8 for all cases in the training stage, Method 1 and Method 2 are in the first rank and the proposed approach and Method 4 are in the second rank. As can be seen in Table 8, the present work is ranked 1 only for Case 4. In other words, the suggested work is better than all methods in detecting the fault level of the rolling element. However, the results of Table 7 illustrate that the accuracy of the technique presented in this paper at the training stage is very close to the results of other methods.

- The accuracy of the proposed method in the final test step for Case 1 and Case 4 is higher than the other methods presented in Table 6. As can been seen in Table 8, the proposed technique is the fourth rank for Case 2 and is the second rank for Case 3. However, the final ranking results in the test phase show that the proposed method and Method 4 are in the first rank and Method 5 is in the second rank.

- The results of the first case indicate that the proposed technique in this work, Method 5 and Method 6 are superior to other methods in identifying defects with very small sizes. In the present approach, using the SWD and OCDET methods, features with very high sensitivity to the presence of defects with very small sizes are generated and selected. In addition, the performance of the current work and other techniques has been tested by Case 1, which includes a variety of bearing defects. As shown in Table 7, the proposed approach has been effective in diagnosing conditions such as healthy bearing, defective inner ring, defective outer ring, and defective ball elements. This result can be related to the informative components obtained by the SWD method, which are used in the FE process of the proposed technique. 
- The results of Table 7 show that the proposed method is more efficient than most methods in detecting different levels of fault in the bearing components such as the inner race, outer race, and rolling element.

- The comparison of the results of the proposed technique with Methods 1, 2, and 4 demonstrates that the hybrid FS method CDET+PSOSCALF is superior to the BPSO algorithm, the Relief-F, and the FDAF-score. This superiority is due to the usage of the PSOSCALF and CDET algorithms in the proposed technique. In the present work, the CDET method has been improved based on increasing the accuracy of the SVM classifier, and this approach is in the category of hybrid methods. In other methods such as 2 and 4, the FS algorithms as Relief-F and FDAF-score play the role of feature pre-selection and removal of the redundant features without regard to increasing the SVM accuracy in the fault detection process. Therefore, it is possible to have less meaningful features in the optimal feature set.

- Comparing the proposed method with Method 2 indicates that the SWD method in extracting the fault-sensitive features is more powerful than the EMD decomposition method.

- The results of Table 7 show that in most cases study, the PSOSCALF algorithm is superior to the BPSO, GMPSO, and GOA algorithms in improving the troubleshooting method. As mentioned in Section 2.5, the PSOSCALF optimization algorithm uses the Levy flight operator and the SCA algorithm update equations to generate responses in the optimization process. The PSOSCALF is designed to generate a variety of responses in the search space by using the Levy flight distribution at the beginning of the optimization process, thus enhancing the exploration phase at this stage. On the other hand, by using the properties of the trigonometric functions of sine and cosine, the exploitation phase is amplified in the next steps. In fact, by balancing the two phases of exploration and exploitation in the optimization process with the help of PSOSCALF, the early convergence and falling into the local optimum are prevented. Such capabilities lead to the better performance of PSOSCALF than the BPSO, GMPSO, and GOA algorithms in the troubleshooting techniques.

- The results show that, unlike most troubleshooting techniques, the values of fault detection accuracy of the present approach in the two stages of training and testing do not differ significantly. In fact, when the present technique is used to analyze a 
specific data set, by observing the results in the training stage, it can be expected that the proposed approach does not predict the final test data too far. On the other hand, for most other methods, a phenomenon similar to over-fitting has occurred. In other words, these methods cannot be expected to predict the status of the final test data as well as the results of the training stage.

Table 7 The results of the proposed method and the other IDF techniques for the CWRU data set.

\begin{tabular}{|c|c|c|c|c|c|}
\hline Case studies & Method & Optimal parameters & $\begin{array}{l}\text { Threshold } \\
\text { value }\end{array}$ & $\begin{array}{l}\text { Average rate of } \\
\text { accuracy in train }(\%)\end{array}$ & $\begin{array}{l}\text { Average rate of } \\
\text { accuracy in test }(\%)\end{array}$ \\
\hline \multirow{7}{*}{$\begin{array}{l}\text { Case 1: fault } \\
\text { identification }\end{array}$} & This study & $C=3,27, \sigma=18.96$ & $\xi=0.50$ & 98.50 & 93.75 \\
\hline & Method 1 & $C=68.58, \sigma=3.14$ & --- & 100 & 88.02 \\
\hline & Method 2 & $C=69.34, \sigma=0.96$ & --- & 100 & 90.93 \\
\hline & Method 3 & $C=31.71, \sigma=1.69$ & $\xi=0.66$ & 98.37 & 90.31 \\
\hline & Method 4 & $C=3.00, \sigma=9.96$ & $\xi=0.28$ & 99.85 & 92.08 \\
\hline & Method 5 & $C=7.72, \sigma=1.36$ & $\xi=0.76$ & 98 & 93.75 \\
\hline & Method 6 & $C=1.81, \sigma=1.72$ & $\xi=0.62$ & 97.67 & 93.75 \\
\hline \multirow{7}{*}{$\begin{array}{l}\text { Case 2: fault level } \\
\text { identification for } \\
\text { inner race }\end{array}$} & This study & $C=2.86, \sigma=12.53$ & $\xi=0.48$ & 98.3889 & 96.44 \\
\hline & Method 1 & $C=63.54, \sigma=4.54$ & --- & 100 & 92.66 \\
\hline & Method 2 & $C=60.62, \sigma=1.25$ & --- & 99.84 & 90.77 \\
\hline & Method 3 & $C=9.99, \sigma=4.66$ & $\xi=0.28$ & 90.13 & 74.00 \\
\hline & Method 4 & $C=2.86, \sigma=7.50$ & $\xi=0.34$ & 99.38 & 98.33 \\
\hline & Method 5 & $C=3.058, \sigma=0.68$ & $\xi=0.21$ & 100 & 96.67 \\
\hline & Method 6 & $C=1.60, \sigma=1.95$ & $\xi=0.17$ & 99.31 & 98.22 \\
\hline \multirow{7}{*}{$\begin{array}{l}\text { Case 3: fault level } \\
\text { identification for } \\
\text { outer race }\end{array}$} & This study & $C=1.66, \sigma=4.20$ & $\xi=0.20$ & 98.50 & 96.56 \\
\hline & Method 1 & $C=66.10, \sigma=3.11$ & --- & 100 & 95.20 \\
\hline & Method 2 & $C=68.35, \sigma=1.92$ & --- & 100 & 84.27 \\
\hline & Method 3 & $C=4.44, \sigma=4.60$ & $\xi=0.14$ & 88.95 & 78.54 \\
\hline & Method 4 & $C=1.96, \sigma=6.43$ & $\xi=0.21$ & 98.29 & 97.60 \\
\hline & Method 5 & $C=29.09, \sigma=2.54$ & $\xi=0.01$ & 79.44 & 71.46 \\
\hline & Method 6 & $C=2.42, \sigma=1.22$ & $\xi=0.17$ & 83.71 & 68.24 \\
\hline \multirow{7}{*}{$\begin{array}{l}\text { Case 4: fault level } \\
\text { identification for } \\
\text { rolling element }\end{array}$} & This study & $C=2.96, \sigma=6.50$ & $\xi=0.49$ & 97.39 & 93.33 \\
\hline & Method 1 & $C=62.87, \sigma=1.43$ & --- & 90.22 & 91.75 \\
\hline & Method 2 & $C=60.95, \sigma=0.88$ & --- & 92.88 & 76.58 \\
\hline & Method 3 & $C=9.46, \sigma=3.77$ & $\xi=0.17$ & 76.49 & 60.16 \\
\hline & Method 4 & $C=3.00, \sigma=6.00$ & $\xi=0.50$ & 97.06 & 84.25 \\
\hline & Method 5 & $C=24.01, \sigma=1.13$ & $\xi=0.41$ & 97.25 & 87.5 \\
\hline & Method 6 & $C=2.57, \sigma=1.02$ & $\xi=0.004$ & 86.12 & 78.42 \\
\hline
\end{tabular}


Table 8 Ranking of the IFD methods in the training and testing stages for the CWRU bearing data set

\begin{tabular}{|c|c|c|c|c|c|c|c|c|}
\hline Step & $\begin{array}{l}\text { Case } \\
\text { study }\end{array}$ & $\begin{array}{l}\text { This } \\
\text { study }\end{array}$ & Method 1 & Method 2 & Method 3 & Method 4 & Method 5 & Method 6 \\
\hline \multirow{4}{*}{ Training } & Case 1 & 3 & 1 & 1 & 4 & 2 & 5 & 6 \\
\hline & Case 2 & 5 & 1 & 2 & 6 & 3 & 1 & 4 \\
\hline & Case 3 & 2 & 1 & 1 & 4 & 3 & 6 & 5 \\
\hline & Case 4 & 1 & 5 & 4 & 7 & 3 & 2 & 6 \\
\hline \multicolumn{2}{|c|}{ Final rank in training } & 2 & 1 & 1 & 4 & 2 & 3 & 4 \\
\hline \multirow{4}{*}{ Testing } & Case 1 & 1 & 5 & 3 & 4 & 2 & 1 & 1 \\
\hline & Case 2 & 4 & 5 & 6 & 7 & 1 & 3 & 2 \\
\hline & Case 3 & 2 & 3 & 4 & 5 & 1 & 6 & 7 \\
\hline & Case 4 & 1 & 2 & 6 & 7 & 4 & 3 & 5 \\
\hline \multicolumn{2}{|c|}{ Final rank in testing } & 1 & 3 & $\overline{4}$ & 5 & 1 & 2 & 3 \\
\hline
\end{tabular}

\subsection{Ahrar Institute of Technology and Higher Education Data Set}

Fig. 17 shows the experimental set of the faults simulator in the rotating machines made by the authors of this paper at Ahrar Institute of Technology and Higher Education (AITHE). These data have been used to evaluate the capability of the IFD method presented in this paper. The components of this setup are demonstrated in Fig. 17. As can be seen, this setup includes an electric motor of $0.5 \mathrm{hp}$, an inverter of $0.5 \mathrm{hp}$ with the ability to change the speed between zero to $60 \mathrm{~Hz}$, a belt-pulley set with a ratio of 2 to 1, a shaft with a length of $50 \mathrm{~cm}$ and diameter of $20 \mathrm{~mm}$, two bearings, two anchor wheels for unbalancing and a data logger. The accelerometer of the AC102-1A series and eight-channel data logger with the maximum sampling frequency of $250 \mathrm{kHz}$ had been used to collect and record the vibration signals. The sensor used in this setup has a sensitivity of $\pm 50 \mathrm{~g}$. The vibration data for different faults were obtained at the sampling rate of $17.85 \mathrm{kHz}$ and the rotational speed of $1900 \mathrm{rpm}$. The bearings used in this system are of $\mathrm{Nu} 204$ ECP type, the geometric specifications of which are presented in Fig. 18. 


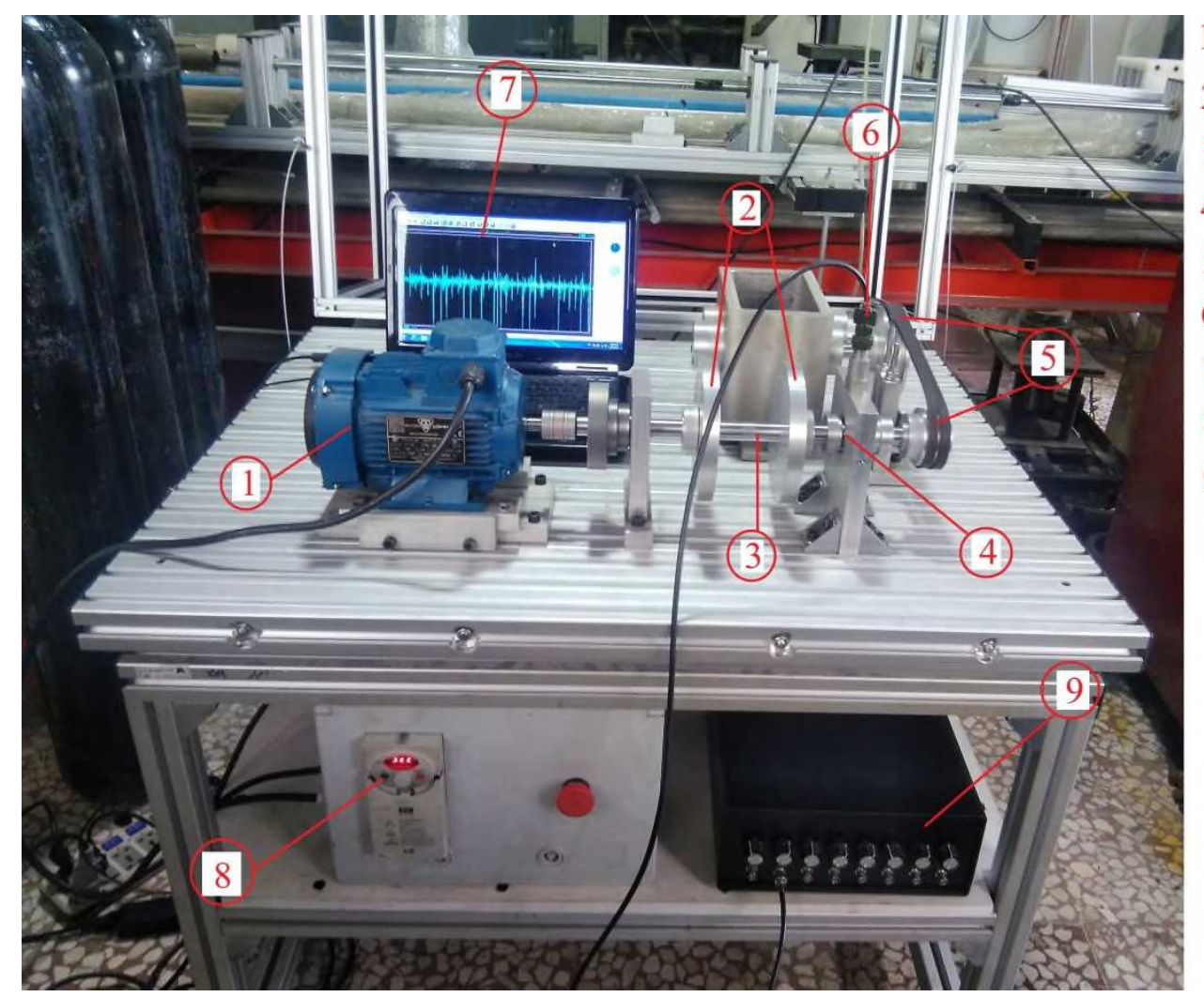

1. Motor

2. Rotors

3. Shaft

4. Bearing

5. Belt Mechanism

6. Accelerometer

7. Laptap

8. Inverter

9. Data Logger

Fig. 17 The fault simulator setup in rotating machine at AITHE

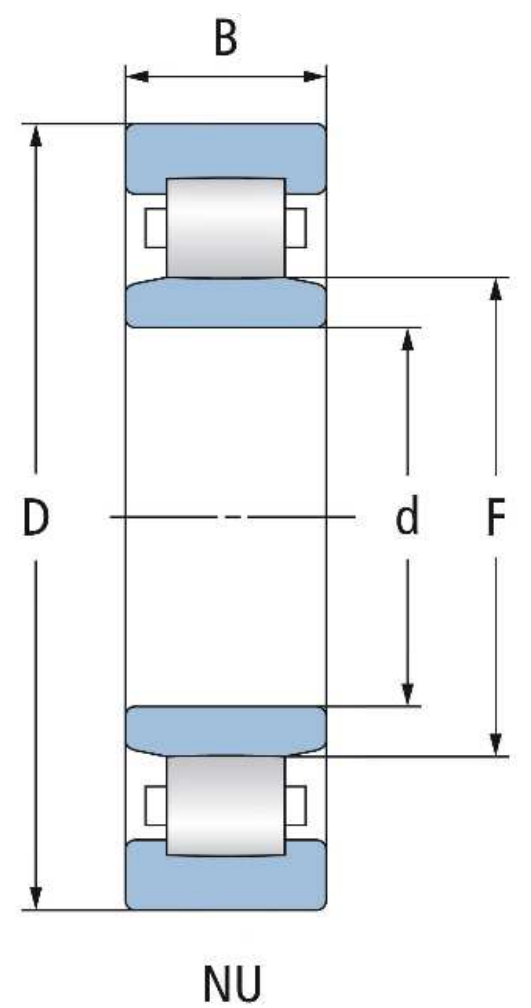

\begin{tabular}{ll}
$\mathbf{d}(\mathrm{mm})$ & 20 \\
\hline $\mathbf{D}(\mathrm{mm})$ & 47 \\
\hline $\mathbf{B}(\mathrm{mm})$ & 14 \\
\hline $\mathbf{F}(\mathrm{mm})$ & 27 \\
\hline Weight in kg & 0,108
\end{tabular}

Fig. 18 Geometric specifications of NU 204 ECP bearing 
The characteristics of the defects investigated in this experiment are described in Table 9. These specifications include class number, type of defect, depth, and width of the defect. The types of faults created in the bearing components are given in Fig. 19. The numbers 1-8 marked in the red circle indicate the defect class. In total, the vibration data were collected for 9 different classes and 20 signals were recorded corresponding to each fault class. The vibration signals corresponding to different fault classes are shown in Fig. 20.

Table 9 The specifications of different fault classes

\begin{tabular}{|c|c|c|c|c|}
\hline Class number & Class type & Fault type & Fault depth $(\mathrm{mm})$ & Fault depth (mm) \\
\hline 1 & Healthy bearing & & & \\
\hline 2 & Faulty inner ring & $\begin{array}{l}\text { A groove with a } \\
\text { small width }\end{array}$ & 1.5 & 1.5 \\
\hline 3 & Faulty inner ring & A wide groove & 1.5 & 3 \\
\hline 4 & Faulty inner ring & $\begin{array}{l}\text { A surface defect } \\
\text { with a small depth }\end{array}$ & 0.3 & 5.5 \\
\hline 5 & $\begin{array}{l}\text { Inner ring with } \\
\text { two fault }\end{array}$ & $\begin{array}{l}\text { Two grooves with } \\
\text { small width }\end{array}$ & 1.5 & 1.5 \\
\hline 6 & Faulty outer ring & A wide groove & 0.3 & 5 \\
\hline 7 & Faulty outer ring & Surface wear & & \\
\hline 8 & $\begin{array}{l}\text { Faulty inner and } \\
\text { outer ring }\end{array}$ & Groove & 1.5 & $\begin{array}{l}1.5 \text { (inner ring) } \\
5 \text { (outer ring) }\end{array}$ \\
\hline 9 & $\begin{array}{l}\text { Rotating } \\
\text { unbalance }\end{array}$ & $\begin{array}{l}\text { Adding a mass to } \\
\text { the rotor }\end{array}$ & & \\
\hline
\end{tabular}



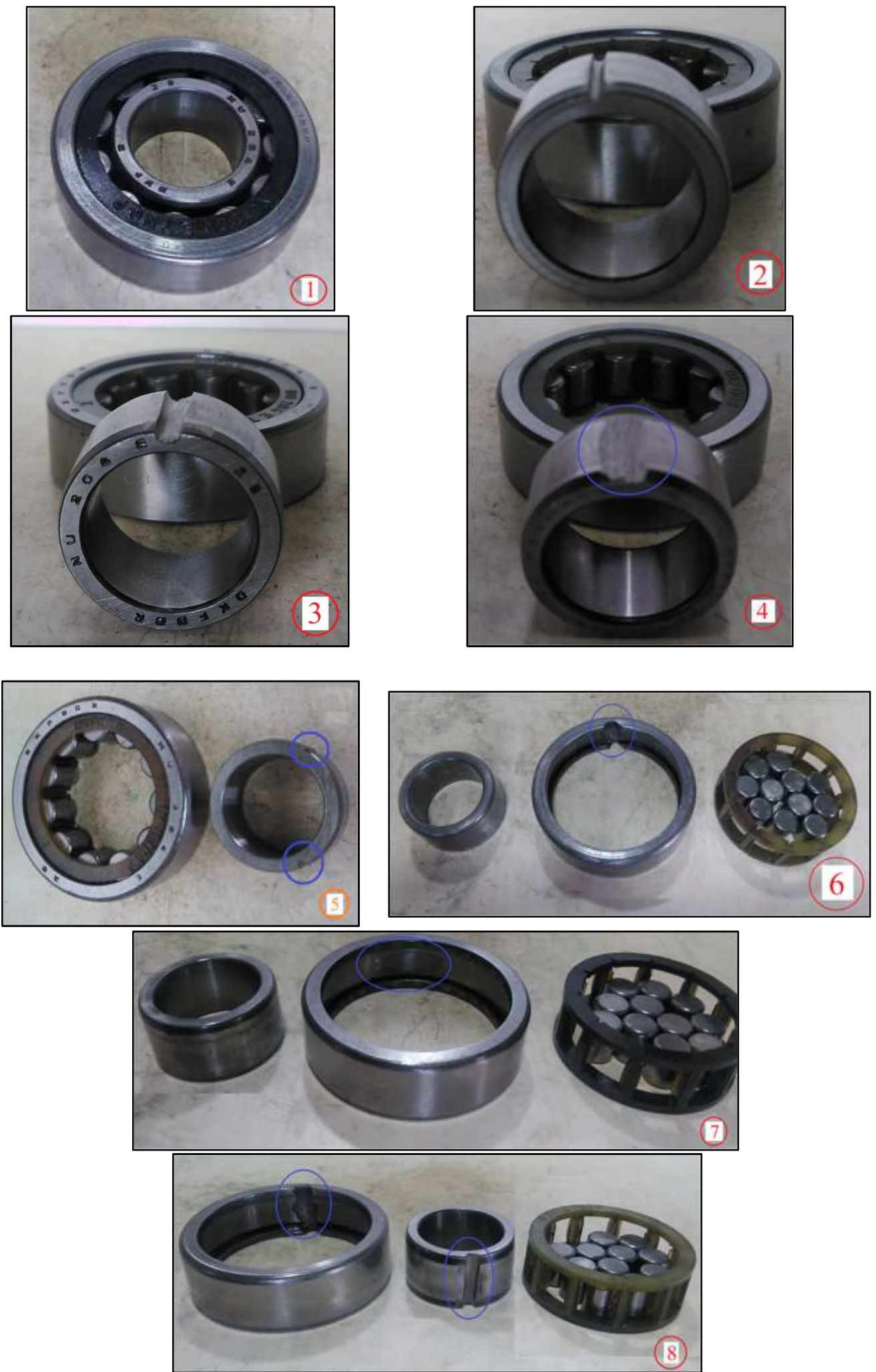

Fig. 19 Types of defects created in the laboratory set of the fault simulator (numbers 1-8 correspond to the class of defects described in Table 8) 

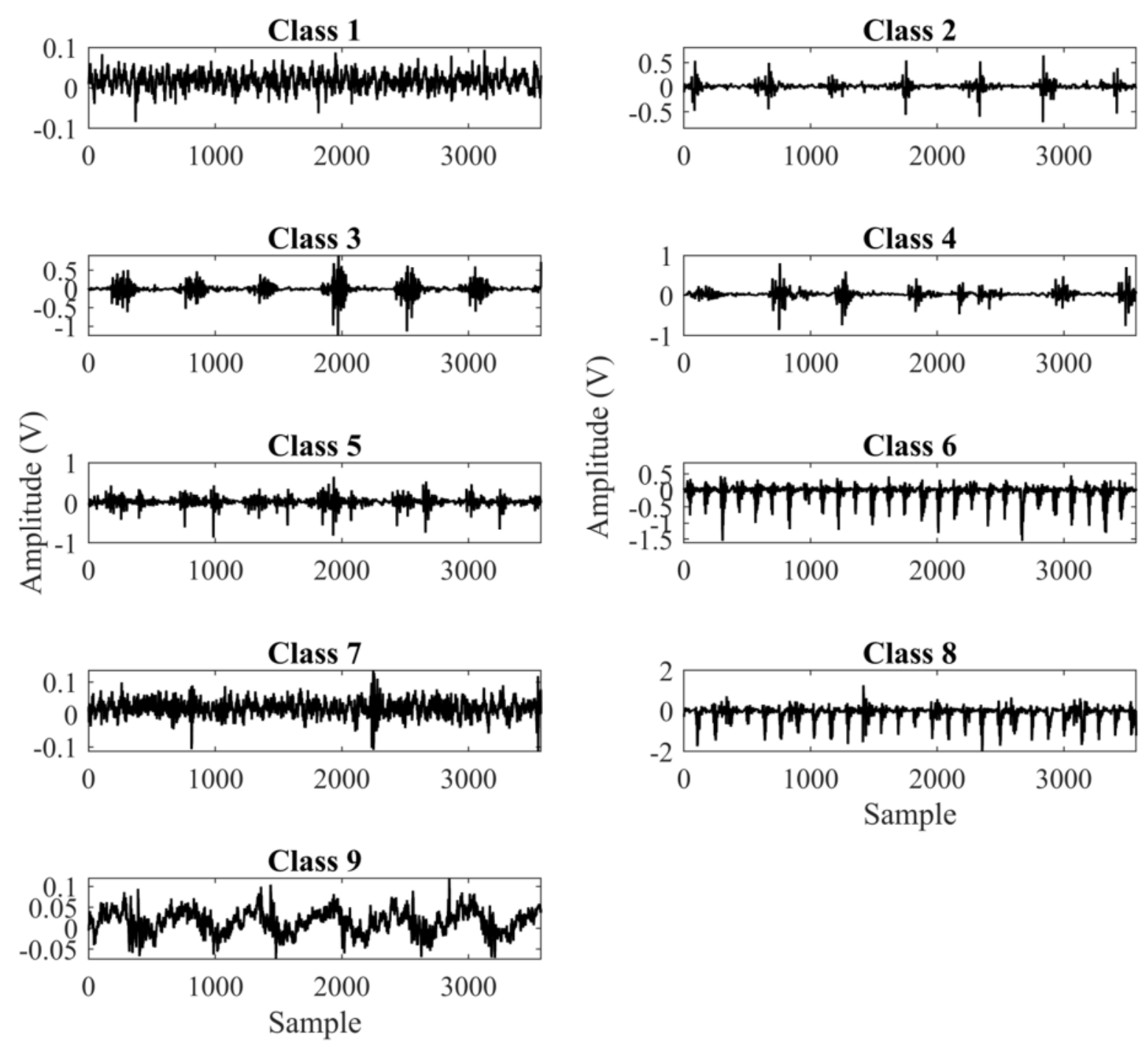

Fig. 20 The vibration signals corresponding to different fault classes

\subsubsection{Results and Discussion}

The time-domain features extracted from the selected OCs have been evaluated by the OCDET technique and the weight of each property is calculated. The results are presented in Fig. 21. Features that have a weight more than the optimal threshold value constitute the optimal feature set. These attributes are marked with «"» in Fig. 21, and their list corresponding to the selected OCs is reported in Table 10. 


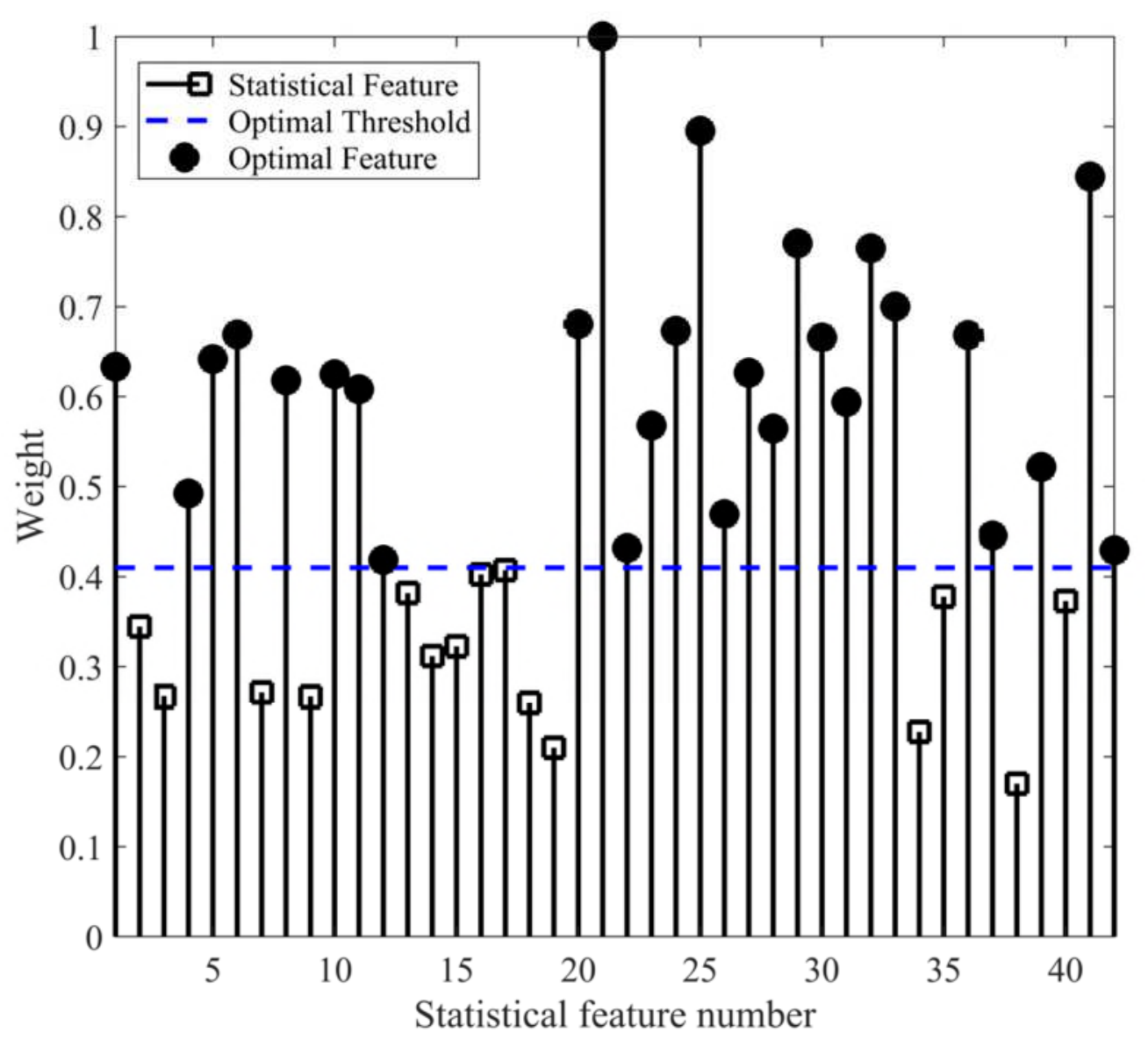

Fig. 21 The weight scores of all features obtained by CDET for AITHE Data Set

Table 10 The best features selected by the CDET for the AITHE data set.

OCs

Optimal features

Mean, Medium, Entropy, Root mean square

The first selected OC

Mean of peaks, Standard deviation

Skewness, EHNR, Teager-Kaiser energy

Mean, Maximum, Minimum, Medium, Energy

Entropy, Range, Root mean square, Peak value

The second selected OC

Mean of peaks, Standard deviation, Skewness

Shape factor, Impulse factor, Hybrid feature 1

Hybrid feature 2, EHNR, Teager-Kaiser energy

Here the presented approach and the other methods described in Table 6 are evaluated using the data acquired in the AITHE experimental set. The results of the implementation of these methods are reported in Table 11. Similar to subsection 5.1.2, each of the techniques 
introduced in Table 6 has been run 30 times independently, and the results are given as the average detection accuracy in the two stages of the training and the final test in Table 11. The optimal SVM parameters and the optimal threshold of the CDET technique are also reported in this table. As shown in Table 11, the value of the optimal threshold parameter obtained by the proposed technique is significantly larger than other methods. In other words, the number of optimal features obtained by the proposed technique is far less than other methods. This result shows that the proposed hybrid technique PSOSCALF+SWD+CDET is more efficient in solving the dimensional problem of the feature space than the FS approaches EMD+PSOSCALF+CDET, SWD+FDAF-score+PSOSCALF，GMPSO+SWD+CDET, and GOA+SWD+CDET.

The accuracy of defect identification results presented in Table 11 demonstrates that Method 3 is better than other methods and the proposed method has the second rank. On the other hand, according to the values of detection accuracy in the final test, the proposed technique and Method 3 in diagnosing the status of the machine and the type of defects have almost the same capability. However, the accuracy of these two methods is significantly higher than other techniques.

Table 11 The results of the proposed method and the other fault detection techniques for the AITHE data set.

\begin{tabular}{llccc}
\hline Method & Optimal parameters & Threshold value & $\begin{array}{c}\text { Average rate of } \\
\text { accuracy in train (\%) }\end{array}$ & $\begin{array}{c}\text { Average rate of } \\
\text { accuracy in test (\%) }\end{array}$ \\
\hline This study & $C=67.14, \sigma=0.81$ & $\xi=0.41$ & 81.80 & 83.01 \\
Method 1 & $C=61.20, \sigma=0.74$ & --- & 67.68 & 62.83 \\
Method 2 & $C=61.20, \sigma=1.62$ & --- & 59.74 & 51.65 \\
Method 3 & $C=80.95, \sigma=1.62$ & $\xi=0.12$ & 95.68 & 88.67 \\
Method 4 & $C=121.26 \sigma=1.28$ & $\xi=0.02$ & 77.64 & 73.11 \\
Method 5 & $C=46.32, \sigma=1.50$ & $\xi=0.30$ & 71.78 & 65.30 \\
Method 6 & $C=2.06, \sigma=1.13$ & $\xi=0.20$ & 66.51 & 60.94 \\
\hline
\end{tabular}

\section{Conclusions}

The main goal of this research is to enhance the hybrid IFD technique in recognizing the bearings defects with variant sizes at different speeds. The proposed method consists of the SWD decomposition algorithm, the CDET method, the SVM classifier, and the PSOSCALF optimization technique. In this way, the CDET procedure is improved by PSOSCALF to increase fault detection accuracy and minimize the number of optimal features. Moreover, to extract the fault-related signatures, the SWD signal processing technique and different 
statistical functions are used. Then, the proposed scheme is applied to the vibration data acquired from the CWRU website and the AITHE experimental setup. The results imply the good performance of the proposed method in identifying the types of faults with variant sizes. In this study, several methods are used to investigate the efficiency of the presented technique. The difference of other methods with the current study is in the implementation of the FD steps such as the signal decomposition, the FS, and the optimization algorithm. The results show that the SWD increases the fault detection accuracy compared with the ITD method. Also, the superiority of OCDET to the Relief-F, the FDAF-score, and the BPSO in producing the most informative features is validated.

\section{Appendix A: the implementation steps of the CDET Method}

Suppose that the feature space of $C$ conditions is as follow:

$\left\{q_{m, c, j}\right\}, \quad m=1,2, \ldots M_{c}, \quad c=1,2, \ldots, C, \quad j=1,2, \ldots, J$

where $q_{m, c, j}$ is the $j^{\text {th }}$ feature value of the $m^{\text {th }}$ sample under the $c^{\text {th }}$ condition, $M_{c}$ the sample number of $c^{\text {th }}$ condition, and $J$ is the feature number of each sample. The process of calculating the weight of features using CDET is as follows:

1. Calculate the average distance of the same condition samples:

$d_{c, j}=\frac{1}{M_{c} \times\left(M_{c}-1\right)} \sum_{l, m=1}^{M_{c}}\left|q_{m, c, j}-q_{l, c, j}\right|, \quad l, m=1,2, \ldots, M_{c}, l \neq m$

Then getting the average distance of $C$ conditions:

$d_{j}^{(w)}=(1 / C) \sum_{C=1}^{C} d_{c, j}$

2. Define and determine the variance factor of $d_{j}^{(w)}$ as follows:

$v_{j}^{(w)}=\max \left(d_{c, j}\right) / \min \left(d_{c, j}\right)$

3. Calculate the average feature values for all samples under the same condition:

$u_{c, j}=\left(1 / M_{c}\right) \sum_{m=1}^{M_{c}} q_{m, c, j}$

Then, evaluate the average distance between various condition samples:

$d_{j}^{(b)}=\frac{1}{C \times(C-1)} \sum_{c, e=1}^{C}\left|u_{e, j}-u_{c, j}\right|, \quad c, e=1,2, \ldots, C, c \neq e$. 
4. Define and calculate the variance factor of $d_{j}^{(b)}$ as follows:

$v_{j}^{(b)}=\frac{\max \left(\left|u_{e, j}-u_{c, j}\right|\right)}{\min \left(\left|u_{e, j}-u_{c, j}\right|\right)}, \quad c, e=1,2, \ldots, C, c \neq e$

5. Determine the compensation factor as follows:

$$
\lambda_{j}=\frac{1}{\frac{v_{j}^{(w)}}{\max \left(v_{j}^{(w)}\right)}+\frac{v_{j}^{(b)}}{\max \left(v_{j}^{(b)}\right)}}
$$

6. The $\bar{\alpha}_{j}$ that is an index for evaluating the $j$-th feature is defined as follows:

$$
\bar{\alpha}_{j}=\frac{\alpha_{j}}{\max \left(\alpha_{j}\right)}
$$

where

$\alpha_{j}=\lambda_{j}\left(d_{j}^{(b)} / d_{j}^{(w)}\right)$

7. The predetermined threshold value, $\xi$, is considered. For all features, the parameter $\xi$ is compared with the distance evaluation criteria. The features with $\overline{\alpha_{J}}>\xi$ is selected as useful attributes, otherwise, is eliminated.

\section{Appendix B: the equations of the PSOSCALF optimization algorithm}

Suppose $\vec{X}_{i}(t)$ and $\vec{V}_{i}(t)$ are the position vector and velocity vector of $i^{\text {th }}$ particle, respectively. The updating equations of the position and velocity of each particle are as follows (Shi 2001):

$$
\begin{aligned}
& \vec{V}_{i}(t+1)=w \vec{V}_{i}(t)+c_{1} r_{1}\left(\vec{X}_{\text {pBest }_{i}}-\vec{X}_{i}(t)\right)+c_{2} r_{2}\left(\vec{X}_{g B e s t}-\vec{X}_{i}(t)\right) \\
& \vec{X}_{i}(t+1)=\vec{X}_{i}(t)+\vec{V}_{i}(t+1)
\end{aligned}
$$

In the above relationships, $\vec{V}_{i}(t+1)$ and $\vec{X}_{i}(t+1)$ are the velocity vector and the position vector at the $t+1$-th moment, respectively. $\vec{X}_{p B e s t}$ and $\vec{X}_{g B e s t}$ are the best personal experience and the best experience of the whole particles, respectively. The random variables $r_{1}$ and $r_{2}$ are chosen in the interval $[0,1]$. The coefficients $c_{1}$ and $c_{2}$ represent the personal learning factor and the global learning factor, respectively. $w$ is the weighting factor that applies the effect of the previous velocity vector at the $t$-th instant in determining the new velocity vector at the $t+1$-th instant. 
In the SCA algorithm, the following relationships are used as the solution updating equations (Mirjalili 2016):

$\vec{X}_{i}(t+1)= \begin{cases}\vec{X}_{i}(t)+z_{1} \sin \left(z_{2}\right)\left|z_{3} P^{t}-\vec{X}_{i}(t)\right|, & z_{4}<0.5 \\ \vec{X}_{i}(t)+z_{1} \cos \left(z_{2}\right)\left|z_{3} P^{t}-\vec{X}_{i}(t)\right|, & z_{4} \geq 0.5\end{cases}$

where the parameters $z_{1}, z_{2}, z_{3}$, and $z_{4}$ represent the key parameters of the SCA method and are set according to Mirjalili (2016). $P^{t}$ is the destination point or the best solution obtained at time $t$. In PSOSCALF, the following combinational equations that benefit from Levy flight and SCA algorithms are used for producing new responses (Nezamivand Chegini et al. 2018):

$\vec{X}_{i}(t+1)= \begin{cases}\operatorname{Levy}_{\text {walk }}\left(\vec{X}_{i}(t)\right)+z_{1} \sin \left(z_{2}\right)\left|z_{3} \vec{X}_{\text {gBest }}-\vec{X}_{i}(t)\right|, & z_{4}<0.5 \\ \text { Levy }_{\text {walk }}\left(\vec{X}_{i}(t)\right)+z_{1} \cos \left(z_{2}\right)\left|z_{3} \vec{X}_{\text {gBest }}-\vec{X}_{i}(t)\right|, & z_{4} \geq 0.5\end{cases}$

The expression of Levy $_{\text {walk }}\left(\vec{X}_{i}(t)\right)$ is the only difference between Eq. (B.4) in PSOSCALF and Eq. (B.3) in SCA which is calculated as follows:

$\operatorname{Levy}_{\text {walk }}\left(\vec{X}_{i}(t)\right)=\vec{X}_{i}(t)+\overrightarrow{\operatorname{step}} \oplus \overrightarrow{\operatorname{random}}\left(\operatorname{size}\left(\vec{X}_{i}(t)\right)\right)$

$\overrightarrow{\text { step }}=$ stepsize $\oplus \vec{X}_{i}(t)$

In the PSOSCALF approach, the relationships presented in Eqs. (B.4 a) and (B.4 b) are added to the process of producing the new position of particles. Moreover, the number of times that the position of a particle does not change is stored in a parameter called the limit value. If this value is greater than or equal to the predetermined value, the position of the particle is calculated by the hybrid relationships in Eq. (B.4).

Details of setting PSOSCALF parameters were presented in (Nezamivand Chegini et al. 2018).

Funding This study was financially supported by the University of Guilan to SNC. The funder had no role in study design, data collection, and analysis, decision to publish or preparation of the manuscript.

\section{Compliance with ethical standards}

Conflict of interest There are no conflicts of interests of this work. 
Authors' contribution SNC and PA designed and coordinated the study. SNC and PA wrote the manuscript. SNC, $\mathrm{AB}, \mathrm{BA}$, and IA reviewed the manuscript and contributed to its revision. All the authors discussed the results and gave their final approved for publication.

\section{References}

Ali JB, Fnaiech N, Saidi L, Chebel-Morello B, Fnaiech F (2015) Application of empirical mode decomposition and artificial neural network for automatic bearing fault diagnosis based on vibration signals. APPL ACOUST. 1(89):16-27. https://doi.org/10.1016/j.apacoust.2014.08.016

Amini Digehsara P, Bagheri A, Moshfegh S (2019) Interval search with quadratic interpolation and stable deviation quantum-behaved particle swarm optimization (IQSQPSO). Int J Multiphysics 13(2): 113-130. 10.21152/1750-9548.13.2.113.

Amini Digehsara P, Nezamivand Chegini S, Bagheri A, Pourabd Roknsaraei M (2020) An improved particle swarm optimization based on the reinforcement of the population initialization phase by scrambled Halton sequence. Cogent Eng 7(1): 1737383. https://doi.org/10.1080/23311916.2020.1737383

Apostolidis GK, Hadjileontiadis LJ (2017) Swarm decomposition: A novel signal analysis using swarm intelligence. Signal Processing 132: 40-50. https://doi.org/10.1016/j.sigpro.2016.09.004

Bearing Data Center (2016) Case Western Reserve University. Available via http://csegroups.case. edu/bearingdatacenter/home.

Bruns A, Fourier-, Hilbert-and wavelet-based signal analysis: are they really different $\begin{array}{lllll}\text { approaches? } & \text { J } & \text { Neurosci } & \text { Methods } & \text { 137(2): }\end{array}$ https://doi.org/10.1016/j.jneumeth.2004.03.002

Chen J, Li Z, Pan J, Chen G, Zi Y, Yuan J, Chen B, He Z (2016) Wavelet transform based on inner product in fault diagnosis of rotating machinery: A review. Mech Syst Signal Process 70:1-35. https://doi.org/10.1016/j.ymssp.2015.08.023

Chih-Wei H, Chih-Jen L (2002) A comparison of methods for multiclass support vector machines. IEEE Trans Neural Netw 13(2): 415-425. 10.1109/72.991427

Cortes C, Vapnik V (1995) Support-Vector Networks. Mach Learn 20(3): 273-297. https://doi.org/10.1007/BF00994018 
Cristianini N, Shawe-Taylor J (2000) An introduction to support vector machines and other kernel-based learning methods. Cambridge university press.

Daubechies I (1990) The wavelet transform, time-frequency localization and signal analysis. IEEE Trans Inf Theory 36(5): 961-1005. 10.1109/18.57199

Deng W, Yao R, Sun M, Zhao H, Luo Y, Dong C (2017) Study on a novel fault diagnosis method based on integrating EMD, fuzzy entropy, improved PSO and SVM. J VIBROENG 19(4):2562-77. https://doi.org/10.21595/jve.2017.18052

Deng W, Zhang S, Zhao H, Yang X (2018) A novel fault diagnosis method based on integrating empirical wavelet transform and fuzzy entropy for motor bearing. IEEE Access 6:35042-56. 10.1109/ACCESS.2018.2834540

Ding J, Xiao D, Li X (2020a) Gear fault diagnosis based on genetic mutation particle swarm optimization VMD and probabilistic neural network algorithm. IEEE Access 21;8:18456-74. doi: 10.1109/ACCESS.2020.2968382.

Ding J, Huang L, Xiao D, Li X (2020b) GMPSO-VMD algorithm and its application to rolling bearing fault feature extraction. Sensors 2020 Jan;20(7):1946. https://doi.org/10.3390/s20071946

Gao C, Wu T, Fu Z (2018) Advanced Rolling Bearing Fault Diagnosis Using Ensemble Empirical Mode Decomposition, Principal Component Analysis and Probabilistic Neural Network. Journal of Robotics, Networking and Artificial Life 5(1): 10-14. https://doi.org/10.2991/jrnal.2018.5.1.3

Gilles J (2013) Empirical wavelet transform. IEEE Trans Signal Process 61(16): 3999-4010. 10.1109/TSP.2013.2265222

Guenther N, Schonlau M (2016) Support Vector Machines. Stata J 16(4): 917-937. $10.1109 / 5254.708428$

Hu Q, He Z, Zhang Z, Zi Y (2007) Fault diagnosis of rotating machinery based on improved wavelet package transform and SVMs ensemble. Mech Syst Signal Process (2): 688-705. https://doi.org/10.1016/j.ymssp.2006.01.007

Huang NE, Shen Z, Long SR, Wu MC, Shih HH, Zheng Q, Yen NC, Tung CC, Liu HH (1998) The empirical mode decomposition and the Hilbert spectrum for nonlinear and nonstationary time series analysis. Proceedings of the Royal Society of London. Series A: mathematical, physical and engineering sciences 454(1971):903-95. 
Jardine AK, Lin D, Banjevic D (2006) A review on machinery diagnostics and prognostics implementing condition-based maintenance. Mech Syst Signal Process 20 (7): 1483-1510. https://doi.org/10.1016/j.ymssp.2005.09.012

Kedadouche M, Thomas M, Tahan A (2016) A comparative study between Empirical Wavelet Transforms and Empirical Mode Decomposition Methods: Application to bearing defect diagnosis. Mech Syst Signal Process 81: 88-107. https://doi.org/10.1016/j.ymssp.2016.02.049

Lei Y, He Z, Zi Y, Chen X (2008) New clustering algorithm-based fault diagnosis using compensation distance evaluation technique. Mech Syst Signal Process 22(2):419-35. https://doi.org/10.1016/j.ymssp.2007.07.013

Liu R, Yang B, Zio E, Chen X (2018) Artificial intelligence for fault diagnosis of rotating machinery: A review. Mech Syst Signal Process 108:33-47. https://doi.org/10.1016/j.ymssp.2018.02.016

Miao Y, Zhao M, Makis V, Lin J (2019) Optimal swarm decomposition with whale optimization algorithm for weak feature extraction from multicomponent modulation signal. Mech Syst Signal Process 22:673-91. https://doi.org/10.1016/j.ymssp.2018.12.034

Mirjalili S (2016) SCA: a sine cosine algorithm for solving optimization problems. Knowledge-Based Systems 96: 120-133. https://doi.org/10.1016/j.knosys.2015.12.022

Nezamivand Chegini S, Bagheri A, Najafi F (2018) PSOSCALF: A new hybrid PSO based on Sine Cosine Algorithm and Levy flight for solving optimization problems. Appl Soft Comput 73: 697-726. https://doi.org/10.1016/j.asoc.2018.09.019

Nezamivand Chegini S, Bagheri A, Najafi F (2019a) Application of a New EWT-Based Denoising Technique in Bearing Fault Diagnosis. Measurement 144: 275-297. https://doi.org/10.1016/j.measurement.2019.05.049

Nezamivand Chegini S, Bagheri A, Najafi F (2019b) A new intelligent fault diagnosis method for bearing in different speeds based on the FDAF-score algorithm, binary particle swarm optimization, and support vector machine. SOFT COMPUT 11: 1-9. https://doi.org/10.1007/s00500-019-04516-z

Pratyay K, Paramita C (2015) Multi-class fault diagnosis of induction motor using Hilbertand Wavelet Transform. Appl Soft Comput 30:341-52. https://doi.org/10.1016/j.asoc.2014.11.062 
Rao A, Kumaresan R (2000) On decomposing speech into modulated components. IEEE Transactions on Speech and Audio Processing 8(3): 240-254. 10.1109/89.841207

Shao K, Fu W, Tan J, Wang K (2021) Coordinated approach fusing time-shift multiscale dispersion entropy and vibrational Harris hawks optimization-based SVM for fault diagnosis $\begin{array}{lllll}\text { of } & \text { rolling } & \text { bearing. } & \text { Measurement } & 173:\end{array}$ https://doi.org/10.1016/j.measurement.2020.108580

Scholkopf, Smola AJ (2001) Learning with kernels: support vector machines, regularization, optimization, and beyond. MIT press.

Shi Y (2001) Particle swarm optimization: developments, applications and resources. in Proceedings of the 2001 congress on evolutionary computation (IEEE Cat. No. 01TH8546). 2001. IEEE. 10.1109/CEC.2001.934374

Teng W, Ding X, Cheng H, Han C, Liu Y, Mu H (2019) Compound faults diagnosis and analysis for a wind turbine gearbox via a novel vibration model and empirical wavelet transform. Renew Energy 136:393-402. https://doi.org/10.1016/j.renene.2018.12.094

Torres ME, Colominas MA, Schlotthauer G, Flandrin P (2011) A complete ensemble empirical mode decomposition with adaptive noise. In2011 IEEE international conference on acoustics, speech and signal processing (ICASSP) 4144-4147. 10.1109/ICASSP.2011.5947265

Vapnik V (2013) The nature of statistical learning theory. Springer science \& business media. Wei J, Huang H, Yao L, Hu Y, Fan Q, Huang D (2020) New imbalanced fault diagnosis framework based on Cluster-MWMOTE and MFO-optimized LS-SVM using limited and complex bearing data. Eng Appl Artif Intell 96: 103966. https://doi.org/10.1016/j.engappai.2020.103966

Widodo A, Yang BS (2007) Support vector machine in machine condition monitoring and fault diagnosis. Mech Syst Signal Process 21(6): 2560-2574. https://doi.org/10.1016/j.ymssp.2006.12.007

Wu Z, Huang NE (2009) Ensemble empirical mode decomposition: a noise-assisted data analysis method. Adv Adapt Data Anal 1(01):1-41.

Xiao D, Ding J, Li X, Huang L (2019) Gear fault diagnosis based on kurtosis criterion VMD and SOM neural network. Appl Sci 9(24):5424. https://doi.org/10.3390/app9245424 
Xu X, Zhao M, Lin J, Lei Y (2016) Envelope harmonic-to-noise ratio for periodic impulses detection and its application to bearing diagnosis. Measurement 91:385-97. https://doi.org/10.1016/j.measurement.2016.05.073

Yang, BS, Kim KJ (2006) Application of Dempster-Shafer theory in fault diagnosis of induction motors using vibration and current signals. Mech Syst Signal Process 20(2): 403420. https://doi.org/10.1016/j.ymssp.2004.10.010

Yang BS, Han T, An JL (2004) ART-KOHONEN neural network for fault diagnosis of rotating machinery. Mech Syst Signal Process 18(3): 645-657. https://doi.org/10.1016/S08883270(03)00073-6

Yan X, Jia M (2019) Application of CSA-VMD and optimal scale morphological slice bispectrum in enhancing outer race fault detection of rolling element bearings. Mech Syst Signal Process 122:56-86. https://doi.org/10.1016/j.ymssp.2018.12.022

Yin H, Qiao J, Fu P, Xia XY (2014) Face feature selection with binary particle swarm optimization and support vector machine. J Inf Hiding Multimed Signal Process 5(4):731-9.

Zhang X, Li C, Wang X, Wu H (2021) A novel fault diagnosis procedure based on improved symplectic geometry mode decomposition and optimized SVM. Measurement 173: 108644. https://doi.org/10.1016/j.measurement.2020.108644

Zhang X, Miao Q, Zhang H, Wang L (2018a) A parameter-adaptive VMD method based on grasshopper optimization algorithm to analyze vibration signals from rotating machinery. Mech Syst Signal Process;108:58-72. https://doi.org/10.1016/j.ymssp.2017.11.029

Zhang X, Zhang Q, Chen M, Sun Y, Qin X, Li H (2018b) A two-stage feature selection and intelligent fault diagnosis method for rotating machinery using hybrid filter and wrapper method. Neurocomputing 275:2426-39. https://doi.org/10.1016/j.neucom.2017.11.016

Zhang X, Liang Y, Zhou J (2015) A novel bearing fault diagnosis model integrated permutation entropy, ensemble empirical mode decomposition and optimized SVM. Measurement 69:164-79. https://doi.org/10.1016/j.measurement.2015.03.017 